\title{
The Spatial Averaging Limit of Covariant Macroscopic Gravity \\ - Scalar Corrections to the Cosmological Equations -
}

\author{
Aseem Paranjape* and T. P. Singh \\ Tata Institute of Fundamental Research, Homi Bhabha Road, \\ Colaba, Mumbai - 400 005, India
}

\begin{abstract}
It is known that any explicit averaging scheme of the type essential for describing the large scale behaviour of the Universe, must necessarily yield corrections to the Einstein equations applied in the Cosmological setting. The question of whether or not the resulting corrections to the Einstein equations are significant, is still a subject of debate, partly due to possible ambiguities in the averaging schemes available. In particular, it has been argued in the literature that the effects of averaging could be gauge artifacts. We apply the formalism of Zalaletdinov's Macroscopic Gravity (MG) which is a fully covariant and nonperturbative averaging scheme, in an attempt to construct gauge independent corrections to the standard Friedmann-Lemaître-Robertson-Walker (FLRW) equations. We find that whereas one cannot escape the problem of dependence on one gauge choice - which is inherent in the assumption of large scale homogeneity and isotropy - it is however possible to construct spacetime scalar corrections to the standard FLRW equations. This partially removes the criticism concerning the corrections being gauge artifacts. For a particular initial choice of gauge which simplifies the formalism, we explicitly construct these scalars in terms of the underlying inhomogeneous geometry, and incidentally demonstrate that the formal structure of the corrections with this gauge choice is identical to that of analogous corrections derived by Buchert in the context of spatial averaging of scalars.
\end{abstract}

\section{INTRODUCTION}

One of the basic assumptions of Cosmology is that the matter distribution in the Universe, when averaged on large enough scales, is homogeneous and isotropic. One then models the metric of spacetime on such large scales as having the Friedmann-Lemaître-RobertsonWalker (FLRW) form, i.e. - having homogeneous and isotropic spatial sections, and applies Einstein's General Relativistic field equations to determine the geometry of the Universe. The idea here is that just like the matter, the metric can also be "averaged" on large scales to yield a highly symmetric geometry. The averaging operation is usually an implicit (and often vague) assumption. It has long been known however [1], that any explicit averaging scheme for the metric of spacetime and energymomentum tensor of matter, must necessarily yield corrections to the Einstein equations - these equations being ideally imposed on length scales where stars (and not galaxies) are pointlike objects.

The problem of constructing an explicit and selfconsistent averaging scheme for the Einstein equations in the Cosmological setting has a long history [2]. Recently, attention has been devoted to an averaging scheme developed by Buchert [3, 4], who defines a spatial averaging operation for scalar quantities within a chosen $3+1$ splitting of spacetime, and derives effective equations which could have nontrivial effects on the assumed, homogeneous and isotropic model of the Universe. The ques-

*Electronic address: aseem@tifr.res.in

†Electronic address: tpsingh@tifr.res.in tion of whether or not the resulting corrections to the Einstein equations are significant, is still a subject of debate [5, 6, 7, 8] and can be considered to be open. Buchert's approach to averaging has also been debated on the grounds that it is concerned with spatial averages, and not spacetime averages.

In such a context, it is perhaps desirable to work with an averaging scheme which starts from a spacetime averaging, and which allows us to construct the corrections to Einstein's equations as physically relevant, gauge independent objects that can (in principle at least) be observationally tested. An averaging scheme for tensors on an arbitrary pseudo-Riemannian manifold has been developed by Zalaletdinov and is detailed in Refs. [9, 10, 11]. This scheme, called Macroscopic Gravity (MG), has been applied to Cosmology in some specific models [12, 13], where the corrections were shown to be of the form of a spatial curvature term in the Friedmann equation. In this paper we address the following question : Can the equations of Zalaletdinov's MG, in a general setting and under suitable assumptions, be brought to a form where the corrections to the FLRW equations can be dealt with in a gauge independent manner? We will show that the issue of a $3+1$ splitting can be reduced to one choice of gauge for the inhomogeneous metric which averages to the FLRW metric in its natural comoving spatial coordinates. While such a choice always needs to be made for any explicit computations (and is indeed inherent in the assumption of large scale homogeneity and isotropy), we will show that it is possible to at least formally construct spacetime scalar quantities which affect the FLRW equations in a nontrivial manner. We will then explicitly construct these scalars assuming that the comoving FLRW spatial sections result on averaging in a particular gauge 
choice which simplifies the formalism, and incidentally also demonstrate that for this gauge choice, the structure of the corrections in Zalaletdinov's MG is formally identical to that of the corrections derived by Buchert [3] (see also Ref. [14]). Further, we will also show that in principle the allowed behaviour (with time) of these corrections is, in principle, more general than of the form of a spatial curvature, as was found in Refs. [12, 13].

We have organized the paper as follows: For the benefit of readers unfamiliar with the details of Macroscopic Gravity, we have devoted considerable space to recalling this formalism in Section II which will also allow us to lay down our notation. Readers may wish to skim this section and go on to Section III, where we refine the assumptions of standard Cosmology and adapt them to the framework of MG, and also discuss the issue of gauge choice. Choosing a particularly convenient gauge for simplicity, we then explicitly compute the corrections to the FLRW equations in Section IV, and then we present the formal results for an arbitrary choice of gauge. This is followed by a comparison between the MG approach and the averaging scheme developed by Buchert, where we present the case that in principle the two approaches are expected to be identical in content, in the sense that they should yield the same physical results. We conclude in Section $\mathrm{V}$ by summarizing and discussing some additional issues.

\section{THE MACROSCOPIC GRAVITY FORMALISM OF ZALALETDINOV}

Our aim in this section is to lay out the notation for the rest of the paper, and to recall the main results and concepts of Zalaletdinov's Macroscopic Gravity (henceforth $\mathrm{MG}$ ). The subject matter we will deal with is of a rather technical nature, and the results are in fact best presented in the language of differential forms. We will therefore begin with a brief recollection of some basic results from differential geometry which will serve to fix the notation. We will then introduce additional notation while describing the results of MG. The latter part of this section will essentially be a repetition of results obtained by Zalaletdinov 9] and Mars and Zalaletdinov [10]. While we shall go through many of the algebraic details in order to make this paper self-contained, we will not go into details of arguments justifying the various assumptions made in the averaging scheme, since our aim here is to apply the formalism developed in Refs. [9, 10, 11].

We shall deal with a differentiable manifold $\mathcal{M}$ endowed with a metric $g_{a b}$ of Lorentzian signature $(-+++)$. We will use lower case Latin characters $a, b, c, \ldots i, j, k \ldots$ to denote spacetime indices taking values $0,1,2,3$; later we will also use upper case Latin characters $A, B, C, \ldots$ to denote spatial indices taking values $1,2,3$ in a chosen $3+1$ splitting of spacetime. We will denote all differential forms and ten- sors on $\mathcal{M}$ by boldface characters. For example, $\boldsymbol{\alpha}=$ $(1 / k !) \alpha_{i_{1} \cdots i_{k}} \mathbf{d} x^{i_{1}} \wedge \cdots \wedge \mathbf{d} x^{i_{k}}$ is a $k$-form written in the coordinate basis of 1 -forms $\left\{\mathbf{d} x^{i}\right\}$, while $\mathbf{v}=\mathbf{e}_{a} v^{a}$ is a vector in the basis $\left\{\mathbf{e}_{a}\right\}$. The connection 1-forms are denoted by $\boldsymbol{\omega}_{i}^{b}=\left(\Gamma_{i j}^{b}\right) \mathbf{d} x^{j}$ where $\Gamma_{i j}^{b}$ are the Christoffel symbols. For a $k$-form $\mathbf{p}_{b \cdots}^{a \cdots}$, we define the "exterior covariant derivative" $\mathbf{D}_{\omega}$ associated with the connection $\boldsymbol{\omega}_{b}^{a}$, as follows :

$$
\mathbf{D}_{\omega} \mathbf{p}_{b \cdots}^{a \cdots}=\mathbf{d p}_{b \cdots}^{a \cdots}-\boldsymbol{\omega}_{b}^{k} \wedge \mathbf{p}_{k \cdots}^{a \cdots}+\boldsymbol{\omega}^{a}{ }_{k} \wedge \mathbf{p}_{b \cdots}^{k \cdots}+\ldots
$$

The compatibility between the metric and the connection on $\mathcal{M}$ is expressed by the condition

$$
\mathbf{D}_{\omega} g_{a b}=\mathbf{d} g_{a b}-g_{a k} \boldsymbol{\omega}_{b}^{k}-g_{b k} \boldsymbol{\omega}_{a}^{k}=0
$$

with a similar condition for the inverse metric $g^{a b}$. The Cartan structure equations are given by

$$
\begin{gathered}
\boldsymbol{\omega}_{b}^{a} \wedge \mathbf{d} x^{b}=0, \\
\mathbf{d} \boldsymbol{\omega}_{b}^{a}+\boldsymbol{\omega}_{c}^{a} \wedge \boldsymbol{\omega}_{b}^{c}=\mathbf{r}_{b}^{a},
\end{gathered}
$$

where $\mathbf{r}_{b}^{a}$ is the curvature 2 -form on $\mathcal{M}$ which defines the Riemann curvature tensor via $\mathbf{r}_{b}^{a}=(1 / 2 !) r_{b c d}^{a} \mathbf{d} x^{c} \wedge \mathbf{d} x^{d}$. Finally, the structure equations (3) and the metric compatibility condition (2) are supplemented by their respective integrability conditions, given by equations (4a), (4b) and (4c)

$$
\begin{gathered}
\mathbf{r}_{b}^{a} \wedge \mathbf{d} x^{b}=0 \\
\mathbf{d r}_{b}^{a}-\omega_{b}^{c} \wedge \mathbf{r}_{c}^{a}+\omega_{c}^{a} \wedge \mathbf{r}_{b}^{c}=\mathbf{D}_{\omega} \mathbf{r}_{b}^{a}=0 \\
g_{a k} \mathbf{r}_{b}^{k}+g_{b k} \mathbf{r}_{a}^{k}=0
\end{gathered}
$$

We will now proceed to recall the main results of MG. The reader is referred to Refs. [9, 10] for details of derivations of the formulae that follow.

The construction of an averaged manifold $\overline{\mathcal{M}}$ from the manifold $\mathcal{M}$ begins with the definition of a bilocal exterior calculus on $\mathcal{M}$, using a bilocal operator $\mathcal{W}_{j}^{a^{\prime}}\left(x^{\prime}, x\right)$. Hereafter, the primed index refers to the point $x^{\prime}$ and the unprimed index to the point $x$, and the bivector $\mathcal{W}_{j}^{a^{\prime}}\left(x^{\prime}, x\right)$ transforms like a vector at $x^{\prime}$ and a covector at $x$. This bivector is assumed to be idempotent [10], i.e. $\mathcal{W}_{c^{\prime \prime}}^{a^{\prime}}\left(x^{\prime}, x^{\prime \prime}\right) \mathcal{W}_{j}^{c^{\prime \prime}}\left(x^{\prime \prime}, x\right)=\mathcal{W}_{j}^{a^{\prime}}\left(x^{\prime}, x\right)$; and to have the coincidence limit $\lim _{x^{\prime} \rightarrow x} \mathcal{W}_{j}^{a^{\prime}}\left(x^{\prime}, x\right)=\delta_{j}^{a}$. This ensures that $\mathcal{W}_{j}^{a^{\prime}}\left(x^{\prime}, x\right)$ has the inverse operator $\mathcal{W}_{j^{\prime}}^{a}\left(x, x^{\prime}\right)$. The bivector $\mathcal{W}_{j}^{a^{\prime}}\left(x^{\prime}, x\right)$ serves three purposes -

(a) It is used to "shift" the exterior derivative by evaluating the derivative at $x^{\prime}$ and antisymmetrizing at $x$ according to the rule (for say, a 2 -form $\boldsymbol{\alpha}$ )

$$
\mathbf{d}_{\mathcal{W}}^{\prime} \boldsymbol{\alpha}^{\prime}=\frac{1}{2 !} \alpha_{a^{\prime} b^{\prime}, c^{\prime}}\left(x^{\prime}\right) \mathcal{W}_{j}^{c^{\prime}}\left(x^{\prime}, x\right) \mathbf{d} x^{j} \wedge \mathbf{d} x^{a^{\prime}} \wedge \mathbf{d} x^{b^{\prime}}
$$


where $\mathbf{d} x^{a^{\prime}}$ is a coordinate basis 1 -form at $x^{\prime}$, and so on. The shifted exterior derivative is used to define a fully bilocal exterior derivative $\mathbf{d}$ as

$$
\mathbf{d}=\mathbf{d}+\mathbf{d}_{\mathcal{W}}^{\prime} .
$$

(b) $\mathcal{W}_{j}^{a^{\prime}}$ is used to define the bilocal extension of tensors and $k$-forms. For example, if $P_{b}^{a}(x)$ is a $(1,1)$ tensor on $\mathcal{M}$, then its bilocal extension is defined as

$$
\widetilde{P}_{b}^{a}\left(x^{\prime}, x\right)=\mathcal{W}_{b}^{b^{\prime}}\left(x^{\prime}, x\right) \mathcal{W}_{a^{\prime}}^{a}\left(x, x^{\prime}\right) P_{b^{\prime}}^{a^{\prime}}\left(x^{\prime}\right)
$$

Similarly, if $\boldsymbol{\alpha}$ is a 2 -form on $\mathcal{M}$, its bilocal extension is defined as

$$
\widetilde{\boldsymbol{\alpha}}\left(x^{\prime}, x\right)=\frac{1}{2 !} \alpha_{a^{\prime} b^{\prime}}\left(x^{\prime}\right) \mathcal{W}_{j}^{a^{\prime}}\left(x^{\prime}, x\right) \mathcal{W}_{k}^{b^{\prime}}\left(x^{\prime}, x\right) \mathbf{d} x^{j} \wedge \mathbf{d} x^{k}
$$

The definitions above can be easily generalised for an arbitrary tensor-valued $k$-form on $\mathcal{M}$. Using the definition of bilocal extensions, the averages of say $P_{b}^{a}$ and $\boldsymbol{\alpha}$ over a spacetime region $\boldsymbol{\Sigma}$ with a supporting point $x$, are then given by

$\bar{P}_{b}^{a}(x)=\left\langle\widetilde{P}_{b}^{a}\right\rangle_{S T}=\frac{1}{V_{\Sigma}} \int_{\Sigma} d^{4} x^{\prime} \sqrt{-g^{\prime}} \widetilde{P}_{b}^{a}\left(x^{\prime}, x\right) ;$
$\overline{\boldsymbol{\alpha}}(x)=\frac{1}{V_{\Sigma}} \int_{\Sigma} d^{4} x^{\prime} \sqrt{-g^{\prime}} \widetilde{\boldsymbol{\alpha}}\left(x^{\prime}, x\right) \quad ; \quad V_{\Sigma}=\int_{\Sigma} d^{4} x^{\prime} \sqrt{-g^{\prime}}$,

the subscript $S T$ standing for 'spacetime'. (Note that $\overline{\boldsymbol{\alpha}}(x)$ is a (local) $k$-form at $x$.) It can be shown [9, 10] that a necessary and sufficient condition for the bilocal exterior derivative to be nilpotent $(\mathbf{d} \mathbf{d}=0)$ and hence for the average of a tensor-valued $k$-form to be a single valued function of the supporting point $x$, is given by

$$
\mathbf{d} \mathbf{W}^{a^{\prime}}=0 ; \mathbf{W}^{a^{\prime}}=\mathcal{W}_{i}^{a^{\prime}} \mathbf{d} x^{i}
$$

and further [10] that a bivector $\mathcal{W}_{j}^{a^{\prime}}$ satisfying all the required properties always exists on an $n$-dimensional differentiable manifold with a volume $n$-form.

(c) Finally, to completely define the exterior derivatives of averaged quantities, $\mathcal{W}_{j}^{a^{\prime}}$ is used to specify a Lie dragging of the averaging region $\boldsymbol{\Sigma}$. This ensures that the volumes of the averaging regions constructed at nearby supporting points are coordinated in a well defined manner. Suppose $x^{a}$ and $x^{a}+\xi^{a} \Delta \lambda$ are the coordinates of two support points, where $\Delta \lambda$ is a small change in the parameter along the integral curve of a given vector field $\xi^{a}$. Symbolically denoting the two points as $x$ and $x+\xi \Delta \lambda$, the averaging region at $x+\xi \Delta \lambda$ is defined in terms of the averaging region $\boldsymbol{\Sigma}$ at $x$, by transporting every point $x^{\prime} \in \boldsymbol{\Sigma}$ around $x$ along the appropriate integral curve of a new bilocal vector field $S^{a^{\prime}}$ defined as $S^{a^{\prime}}\left(x^{\prime}, x\right)=\mathcal{W}_{j}^{a^{\prime}}\left(x^{\prime}, x\right) \xi^{j}(x)$, and thereby constructing the averaging region $\boldsymbol{\Sigma}(\Delta \lambda)$ with support point $x+\xi \Delta \lambda$. (See Refs. [9, 10] for further discussion on the significance of this averaging region coordination.) With this definition, the exterior derivative of the average of a $k$-form $\mathbf{p}_{b}^{a}$ can be written as [9]

$$
\mathbf{d} \overline{\mathbf{p}}_{b}^{a}=\left\langle\mathbf{d} \widetilde{\mathbf{p}}_{b}^{a}\right\rangle_{S T}+\left\langle\operatorname{div}_{\epsilon} \mathbf{W} \wedge \widetilde{\mathbf{p}}_{b}^{a}\right\rangle_{S T}-\left\langle\operatorname{div}_{\epsilon} \mathbf{W}\right\rangle_{S T} \wedge \overline{\mathbf{p}}_{b}^{a}
$$

where we have defined, in keeping with the notation of Ref. [9],

$$
\begin{aligned}
\operatorname{div}_{\epsilon} \mathbf{W} & =\mathcal{W}_{j: a^{\prime}}^{a^{\prime}} \mathbf{d} x^{j} \\
& \equiv\left(\mathcal{W}_{j, a^{\prime}}^{a^{\prime}}+\mathcal{W}_{j}^{a^{\prime}} \partial_{a^{\prime}} \ln \sqrt{-g^{\prime}}\right) \mathbf{d} x^{j} .
\end{aligned}
$$

Clearly, it is desirable to choose a coordination bivector $\mathcal{W}_{j}^{a^{\prime}}$ which satisfies

$$
\operatorname{div}_{\epsilon} \mathbf{W}=0
$$

since firstly, this allows us to commute the exterior derivative with the averaging according to

$$
\mathbf{d} \overline{\mathbf{p}}_{b}^{a}=\left\langle\mathbf{d} \widetilde{\mathbf{p}}_{b}^{a}\right\rangle_{S T},
$$

and secondly, it implies that the volume of the averaging region is held constant during the coordination [10], and is therefore a free parameter in the formalism. It can be shown [10] that firstly, the general solution of Eqn. (10) for an idempotent coordination bivector is given by

$$
\mathcal{W}_{j}^{a^{\prime}}\left(x^{\prime}, x\right)=f_{m}^{a^{\prime}}\left(x^{\prime}\right) f_{j}^{-1 m}(x),
$$

where $f_{m}^{a}(x) \boldsymbol{\partial}_{a}=\mathbf{f}_{m}$ is any vector basis satisfying the commutation relations

$$
\left[\mathbf{f}_{i}, \mathbf{f}_{j}\right]=C_{i j}^{k} \mathbf{f}_{k} ; C_{i j}^{k}=\text { constant }
$$

and secondly, that Eqn. (13) with the coordination bivector given by Eqn. (15) is always integrable on a differentiable manifold with a given volume $n$-form. Further, for the special class of bivectors for which $C_{i j}^{k} \equiv 0$, the vectors $\left\{\mathbf{f}_{k}\right\}$ form a coordinate basis, with 'proper' coordinate functions $\phi^{m}(x)$ say, so that

$$
f_{m}^{a}\left(x\left(\phi^{n}\right)\right)=\frac{\partial x^{a}}{\partial \phi^{m}} \quad ; \quad f_{j}^{-1 m}\left(\phi\left(x^{k}\right)\right)=\frac{\partial \phi^{m}}{\partial x^{j}},
$$

and satisfying Eqn. (13) makes this proper coordinate system volume preserving, with $g\left(\phi^{m}\right)=$ constant. When expressed in terms of such a volume preserving coordinate (VPC) system, the coordination bivector takes its most simple form, namely

$$
\left.\mathcal{W}_{j}^{a^{\prime}}\left(x^{\prime}, x\right)\right|_{\text {proper }}=\delta_{j}^{a^{\prime}}
$$

Volume preserving coordinates in fact form a large class in themselves, generalizing the Cartesian coordinate system of Minkowski spacetime. For a discussion on the properties of VPCs and the associated bivectors $\mathcal{W}_{a}^{a^{\prime}}$, see Sec. 8 of Ref. [10]. We now turn to describing the geometry on a manifold $\overline{\mathcal{M}}$ which is to be considered an 
averaged version of the manifold $\mathcal{M}$. We denote the bilocal extension of the connection 1 -form $\boldsymbol{\omega}^{a}{ }_{b}$ on $\mathcal{M}$, as $\boldsymbol{\Omega}^{a}{ }_{b}$, so that

$\boldsymbol{\Omega}^{a}{ }_{b}\left(x^{\prime}, x\right)=\Gamma_{b^{\prime} c^{\prime}}^{a^{\prime}}\left(x^{\prime}\right) \mathcal{W}_{a^{\prime}}^{a}\left(x, x^{\prime}\right) \mathcal{W}_{b}^{b^{\prime}}\left(x^{\prime}, x\right) \mathcal{W}_{c}^{c^{\prime}}\left(x^{\prime}, x\right) \mathbf{d} x^{c}$.

The key idea of MG is that the average $\overline{\mathbf{\Omega}}^{a}{ }_{b}$ of the connection 1 -form on $\mathcal{M}$, is to be considered the connection 1 -form on the averaged manifold $\overline{\mathcal{M}}$. The goal is then to average out the bilocal extensions of the structure equations (3) and the compatibility condition (2) and their integrability conditions (4), and to express them in terms of appropriate differential forms defined on $\overline{\mathcal{M}}$. The bilocal extensions of Eqns. (3) and (2), are respectively given by

$$
\begin{gathered}
\boldsymbol{\Omega}^{a}{ }_{b} \wedge \mathbf{d} x^{b}=0, \\
\mathbf{d} \boldsymbol{\Omega}_{b}^{a}+\boldsymbol{\Omega}^{a}{ }_{c} \wedge \boldsymbol{\Omega}^{c}{ }_{b}=\widetilde{\mathbf{r}}_{b}^{a}, \\
\mathbf{D}_{\Omega} \widetilde{g}_{a b}=\mathbf{d} \widetilde{g}_{a b}-\widetilde{g}_{a k} \boldsymbol{\Omega}^{k}{ }_{b}-\widetilde{g}_{b k} \boldsymbol{\Omega}^{k}{ }_{a}=0,
\end{gathered}
$$

where, in the last equation, $\mathbf{D}_{\Omega}$ is the bilocal covariant exterior derivative associated with the bilocal connection $\boldsymbol{\Omega}^{a}{ }_{b}$. The integrability conditions of Eqns. (20) are given by the bilocal extensions of Eqns. (4),

$$
\begin{aligned}
& \widetilde{\mathbf{r}}^{a}{ }_{b} \wedge \mathbf{d} x^{b}=0, \\
& \mathbf{d} \widetilde{\mathbf{r}}^{a}{ }_{b}-\boldsymbol{\Omega}^{c}{ }_{b} \wedge \widetilde{\mathbf{r}}^{a}{ }_{c}+\boldsymbol{\Omega}^{a}{ }_{c} \wedge \widetilde{\mathbf{r}}_{b}{ }_{b}=0, \\
& \widetilde{g}_{a k} \widetilde{\mathbf{r}}_{b}^{k}+\widetilde{g}_{b k} \widetilde{\mathbf{r}}_{a}^{k}=0 .
\end{aligned}
$$

To proceed with the averaging, a correlation 2 -form is defined

$$
\mathbf{Z}_{b j}^{a i}=\left\langle\boldsymbol{\Omega}_{b}^{a} \wedge \boldsymbol{\Omega}^{i}{ }_{j}\right\rangle_{S T}-\overline{\boldsymbol{\Omega}}_{{ }_{b}}^{a} \wedge \overline{\boldsymbol{\Omega}}^{i}{ }_{j}
$$

The average of the curvature 2 -form $\mathbf{r}^{a}{ }_{b}$ on $\mathcal{M}$ is denoted $\mathbf{R}^{a}{ }_{b} \equiv\left\langle\widetilde{\mathbf{r}}^{a}{ }_{b}\right\rangle_{S T}$, and the curvature 2-form on the averaged manifold $\overline{\mathcal{M}}$ is denoted $\mathbf{M}^{a}{ }_{b}$,

$$
\mathbf{M}_{b}^{a}=\mathbf{d} \overline{\boldsymbol{\Omega}}^{a}{ }_{b}+\overline{\boldsymbol{\Omega}}^{a}{ }_{k} \wedge \overline{\boldsymbol{\Omega}}_{b}^{k} .
$$

Equations (20a) and (20b) then average out to give

$$
\begin{gathered}
\overline{\boldsymbol{\Omega}}^{a}{ }_{c} \wedge \mathbf{d} x^{c}=0, \\
\mathbf{M}_{b}^{a}=\mathbf{R}_{b}^{a}-\mathbf{Z}_{k b}^{a k} .
\end{gathered}
$$

It can further be shown [9] that the algebraic identities of the curvature 2 -form on $\overline{\mathcal{M}}$ hold :

$$
\mathbf{M}_{c}^{a} \wedge \mathbf{d} x^{c}=0 ; \quad \mathbf{M}_{a}^{a}=0,
$$

To average out Eqn. (21b), one needs to introduce a correlation 3-form which fixes the differential properties of the 2-form $\mathbf{Z}_{b j}^{a}{ }_{b j}$. The differential properties of this
3 -form are then fixed by introducing a 4-form. However, accounting for these additional correlation forms is extremely complicated. We will therefore make the simpler assumption (consistent with the formalism) of setting the correlation 3 -form and 4-form to zero, with the condition

$$
\mathbf{D}_{\bar{\Omega}} \mathbf{Z}_{b j}^{a}{ }_{b j}=0
$$

It will be an interesting exercise to check whether this assumption is, in fact, justified in case of averaging an exact inhomogeneous model. Eqn. (26) has the integrability condition [9]

$$
\mathbb{P}\left(\mathbf{R}_{c}^{a} \wedge \mathbf{Z}_{b j}^{c i}-\mathbf{Z}_{b k}^{a i} \wedge \mathbf{R}^{k}{ }_{j}\right)=0 .
$$

Here the symbol $\mathbb{P}$ permutes the free indices in, say $\mathbf{K}_{b j n}^{a i m}$ pairwise according to $\mathbb{P}\left(\mathbf{K}_{b j n}^{a i m}\right)=$

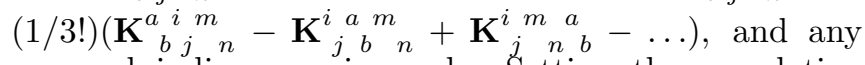
summed indices are ignored. Setting the correlation 3 -form and 4-form to zero also imposes the condition [11, 12 ]

$$
\mathbb{P}\left(\mathbf{Z}_{b{ }_{b}{ }^{c}} \wedge \mathbf{Z}_{i k}^{d j}\right)=0
$$

Eqn. (21b) averages out to give the Bianchi identities for the curvature 2 -form on $\overline{\mathcal{M}}$

$$
\mathbf{D}_{\bar{\Omega}} \mathbf{M}_{b}^{a}=\mathbf{d M}_{b}^{a}-\overline{\mathbf{\Omega}}_{b}^{k} \wedge \mathbf{M}_{k}^{a}+\overline{\mathbf{\Omega}}^{a}{ }_{k} \wedge \mathbf{M}_{b}^{k}=0 .
$$

To average out equations (20c) and (21c), one needs to make additional assumptions. For a class of slowly varying tensor fields (tensor-valued $k$-forms) $\mathbf{c}_{n \cdots}^{m \cdots}$ on $\mathcal{M}$ such as the metric and other covariantly constant tensors, and Killing tensors, etc., if one assumes that

$$
\begin{gathered}
\left\langle\boldsymbol{\Omega}_{b}^{a} \wedge \widetilde{\mathbf{c}}_{n \cdots}^{m \cdots}\right\rangle_{S T}=\overline{\boldsymbol{\Omega}}_{b}^{a} \wedge \overline{\mathbf{c}}_{n \cdots}^{m \cdots}, \\
\left\langle\boldsymbol{\Omega}^{a}{ }_{b} \wedge \boldsymbol{\Omega}^{i}{ }_{j} \wedge \widetilde{\mathbf{c}}_{n \cdots}^{m \cdots}\right\rangle_{S T}=\left\langle\boldsymbol{\Omega}^{a}{ }_{b} \wedge \boldsymbol{\Omega}_{j}^{i}\right\rangle_{S T} \wedge \overline{\mathbf{c}}_{n \cdots}^{m \cdots},
\end{gathered}
$$

then Eqn. (20c) (and its analogue for $g^{a b}$ ) average out to give

$$
\mathbf{D}_{\bar{\Omega}} \bar{g}_{a b}=0 ; \quad \mathbf{D}_{\bar{\Omega}} \bar{g}^{a b}=0 \text {. }
$$

Further, for a general slowly varying object $\mathbf{c}_{n \cdots}^{m \cdots}$, the following identity holds

$$
\begin{aligned}
& \left\langle\widetilde{\mathbf{r}}^{a}{ }_{b} \wedge \widetilde{\mathbf{c}}_{n \cdots}^{m \cdots}\right\rangle_{S T}-\mathbf{R}^{a}{ }_{b} \wedge \overline{\mathbf{c}}_{n \cdots}^{m \cdots} \\
& -\left\langle\boldsymbol{\Omega}^{a}{ }_{b} \wedge \mathbf{D}_{\Omega} \widetilde{\mathbf{c}}_{n \cdots}^{m \cdots}\right\rangle_{S T}+\overline{\boldsymbol{\Omega}}^{a}{ }_{b} \wedge \mathbf{D}_{\bar{\Omega}} \overline{\mathbf{c}}_{n \cdots}^{m \cdots} \\
& =-\mathbf{Z}^{a}{ }_{b}{ }^{m}{ }_{j} \wedge \overline{\mathbf{c}}_{n \cdots}^{j \cdots}-\ldots+\mathbf{Z}^{a}{ }_{b}{ }^{j}{ }_{n} \wedge \mathbf{c}_{j \cdots}^{m \cdots}+\ldots,
\end{aligned}
$$

which averages out Eqn. (21c) (and its analogue for $g^{a b}$ ) to give

$$
\bar{g}_{a k} \mathbf{M}_{b}^{k}+\bar{g}_{k b} \mathbf{M}_{a}^{k}=0 ; \mathbf{M}_{k}^{a} \bar{g}^{k b}+\mathbf{M}_{k}^{a} \bar{g}^{k b}=0 .
$$

Eqn. (31) allows one to choose $G_{a b}=\bar{g}_{a b}$, where $G_{a b}$ is the metric on the averaged manifold $\overline{\mathcal{M}}$. In general 
however, we have $G^{a b} \neq \bar{g}^{a b}$, and one defines the tensor $U^{a b} \equiv \bar{g}^{a b}-G^{a b}$ to keep track of this difference. (See Ref. 9] for details.) However, we show in Appendix (A 1) that when the averaged manifold is highly symmetric, as in the case of a manifold with homogeneous and isotropic spatial sections which we will consider, one finds that $U^{a b}=0$ (see also the last paper in Ref. [11]). In the general case, it turns out that Eqn. (32) is all that is needed to average out the Einstein equations

$$
g^{a k} r_{k b}-\frac{1}{2} \delta_{b}^{a} g^{i j} r_{i j}=-\kappa t_{b}^{a(\mathrm{mic})},
$$

where $\kappa=8 \pi G_{N}, t_{b}^{a(\text { mic })}$ is the microscopic energy momentum tensor of the matter distribution, and the Ricci tensor $r_{a b}$ on $\mathcal{M}$ is defined according to the sign convention $r_{a b}=r_{a b j}^{j}$. The averaging leads to the equations

$$
\begin{aligned}
G^{a k} M_{k b}-\frac{1}{2} \delta_{b}^{a} G^{i j} M_{i j} & =-\kappa\left\langle\widetilde{t}_{b}^{a(\mathrm{mic})}\right\rangle_{S T} \\
& +\left(Z^{a}{ }_{i j b}-\frac{1}{2} \delta_{b}^{a} Z^{k}{ }_{i j k}\right) \bar{g}^{i j} \\
& -\left(U^{a k} M_{k b}-\frac{1}{2} \delta_{b}^{a} U^{i j} M_{i j}\right),
\end{aligned}
$$

where $M_{a b}=M_{a b j}^{j}$ is the Ricci tensor on $\overline{\mathcal{M}}$ and we have defined

$$
Z^{a}{ }_{i j b}=2 Z_{i k j b}^{a}{ }^{k} \quad ; \quad \mathbf{Z}_{b j}^{a}{ }_{b j}=Z_{b m j n}^{a} \mathbf{d} x^{m} \wedge \mathbf{d} x^{n} .
$$

The averaged equations (35) differ from the usual Einstein equations by the correlation tensor which we define as

$C_{b}^{a}=\left(Z^{a}{ }_{i j b}-\frac{1}{2} \delta_{b}^{a} Z^{m}{ }_{i j m}\right) \bar{g}^{i j}-\left(U^{a k} M_{k b}-\frac{1}{2} \delta_{b}^{a} U^{i j} M_{i j}\right)$.

Hence, denoting the Einstein tensor on $\overline{\mathcal{M}}$ as $E_{b}^{a}$, and defining the tensor $T_{b}^{a}$ via

$$
T_{b}^{a}=\left\langle\widetilde{t}_{b}^{a(\mathrm{mic})}\right\rangle_{S T}
$$

the averaged Einstein equations read

$$
E_{b}^{a}=-\kappa T_{b}^{a}+C_{b}^{a}
$$

Since the left hand side of Eqn. (39) is covariantly conserved by construction $\left(E_{b ; a}^{a}=0\right)$, where the semicolon denotes covariant differentiation with respect to the connection on $\overline{\mathcal{M}}$, in general one has

$$
\left(-\kappa T_{b}^{a}+C_{b}^{a}\right)_{; a}=0
$$

with no condition on $T_{b}^{a}$ and $C_{b}^{a}$ separately. However, the condition (26) after taking appropriate traces, reduces to

$$
C_{b ; a}^{a}=0
$$

(see the second of Ref. [11]) which implies that the averaged energy-momentum tensor $T_{b}^{a}$ is also covariantly conserved.

It can also be shown that in 4 dimensions, the $720 a$ priori independent components of $Z^{a}{ }_{b m}{ }^{i}{ }_{j n}$ are subject to 680 constraints arising from Eqns. (27) and (28). This leaves 40 independent components which combine to give the 10 independent components of the correlation tensor $C_{b}^{a}$. The conditions in Eqns. (27) and (28) do not constrain the components of $C_{b}^{a}$, which follows from considering the structure of those equations. (See also Ref. [12].)

\section{A $3+1$ SPACETIME SPLITTING AND THE SPATIAL AVERAGING LIMIT}

We are now in a position to apply the $\mathrm{MG}$ formalism to the problem of Cosmology. We start with the assumption that Einstein's equations are to be imposed on length scales where stars are pointlike objects (we denote such a scale as $L_{\text {inhom }}$ ). The averaging we perform will be directly at a length scale $L_{\text {FLRW }}$ larger than about $100 h^{-1} \mathrm{Mpc}$ or so. This averaging scale is assumed to satisfy $L_{\text {inhom }} \ll L_{\text {FLRW }} \ll L_{\text {Hubble }}$ where $L_{\text {Hubble }}$ is the length scale of the observable universe. The averaging will be assumed to yield a geometry which has homogeneous and isotropic spatial sections. In other words, we will assume that the averaged manifold $\overline{\mathcal{M}}$ admits a preferred, hypersurface-orthogonal unit timelike vector field $\bar{v}^{a}$, which defines 3-dimensional spacelike hypersurfaces of constant curvature, and that $\bar{v}^{a}$ is tangent to the trajectories of observers who see an isotropic Cosmic Background Radiation. (These "observers" are defined in the averaged manifold - we will clarify below what they correspond to in the inhomogeneous manifold.) Throughout the rest of this paper, for simplicity, we will work with the special case where the spatial sections on $\overline{\mathcal{M}}$ defined by $\bar{v}^{a}$ are flat. (In principle the entire calculation can be repeated for non-flat spatial sections as well.) One can then choose coordinates $\left(t, x^{A}\right), A=1,2,3$, on $\overline{\mathcal{M}}$ such that the spatial line element takes the form

$$
{ }^{(\overline{\mathcal{M}})} d s_{\text {spatial }}^{2}=a^{2}(t) \delta_{A B} d x^{A} d x^{B},
$$

where $\delta_{A B}=1$ for $A=B$, and 0 otherwise, and we have $\bar{v}^{a}=\left(\bar{v}^{t}, 0,0,0\right)$ so that the spatial coordinates are comoving with the preferred observers. The vector field $\bar{v}^{a}$ also defines a proper time (the cosmic time) $\tau$ such that $\partial_{\tau}=\bar{v}^{a} \partial_{a}=\bar{v}^{t} \partial_{t}$. We will further assume that the averaged energy-momentum tensor $T_{b}^{a}$ can be written in the form of a perfect fluid, as

$$
T_{b}^{a}=\rho \bar{v}^{a} \bar{v}_{b}+p \pi_{b}^{a}
$$

where the projection operator $\pi_{b}^{a}$ is defined as

$$
\pi_{b}^{a}=\delta_{b}^{a}+\bar{v}^{a} \bar{v}_{b}
$$


and $\rho$ and $p$ are the homogeneous energy density and pressure respectively, as measured by observers moving on trajectories (in $\overline{\mathcal{M}}$ ) with the tangent vector field $\bar{v}^{a}$,

$$
\rho \equiv T_{b}^{a} \bar{v}^{b} \bar{v}_{a} \quad ; \quad p \equiv \frac{1}{3} \pi_{a}^{b} T_{b}^{a}
$$

$\rho$ and $p$ are observationally relevant quantities, since all measurements of the matter energy density, especially those from studies of Large Scale Structure, interpret observations in the context of the averaged geometry. (See Refs. [17, 18] for more careful treatments of this point.) An important consequence of the above assumptions is that the correlation tensor $C_{b}^{a}$, when expressed in terms of the natural coordinates adapted to the spatial sections defined by the vector field $\bar{v}^{a}$, is spatially homogeneous. This is clear when the modified Einstein equations (39) are written in these natural coordinates.

The existence of the vector field $\bar{v}^{a}$ with the attendant assumptions described above, allows us to separate out the nontrivial components of the (FLRW) Einstein tensor $E_{b}^{a}$ on $\overline{\mathcal{M}}$ in a coordinate independent fashion - the Einstein tensor can be written as

$$
\begin{aligned}
E_{b}^{a} & =j_{1}(x) \bar{v}^{a} \bar{v}_{b}+j_{2}(x) \pi_{b}^{a} \\
j_{1}(x) & \equiv E_{b}^{a} \bar{v}^{b} \bar{v}_{a} \quad ; \quad j_{2}(x) \equiv \frac{1}{3}\left(\pi_{a}^{b} E_{b}^{a}\right),
\end{aligned}
$$

where $j_{1}(x)$ and $j_{2}(x)$ are scalar functions whose form depends upon the coordinates used. The remaining components given by $\pi_{k}^{b} E_{b}^{a} \bar{v}_{a}$ and the traceless part of $\pi_{a}^{i} \pi_{k}^{b} E_{b}^{a}$, vanish identically. Since the energy-momentum tensor $T_{b}^{a}$ in Eqn. (43) also has an identical structure, this structure is therefore also imposed on the correlation tensor $C_{b}^{a}$. Namely, $\pi_{k}^{b} C_{b}^{a} \bar{v}_{a}$ and the traceless part of $\pi_{a}^{i} \pi_{k}^{b} C_{b}^{a}$ must vanish. This is a condition on the underlying inhomogeneous geometry, irrespective of the coordinates used on either $\mathcal{M}$ or $\overline{\mathcal{M}}$, and is clearly a consequence of demanding that the averaged geometry have the symmetries of the FLRW spacetime. (Alternatively, one could model the averaged energy-momentum tensor as having some anisotropic components, which would then have to be balanced by the corresponding components of the correlation tensor. We will not pursue this idea here, and will restrict ourselves to the more conservative assumption of a homogeneous and isotropic averaged matter distribution (43).)

This leads us to the crucial question of the choice of gauge for the underlying geometry : namely, what choice of spatial sections for the inhomogeneous geometry, will lead to the spatial sections of the FLRW metric in the comoving coordinates defined in Eqn. (42)? Since the matter distribution at scale $L_{\text {inhom }}$ need not be pressurefree (or, indeed, even of the perfect fluid form), there is clearly no natural choice of gauge available, although locally, a synchronous reference frame can always be chosen. We note that there must be at least one choice of gauge in which the averaged metric has spatial sections in the form (42) - this is simply a refinement of the Cosmological Principle, and of the Weyl postulate, according to which the Universe is homogeneous and isotropic on large scales, and individual galaxies are considered as the "observers" travelling on trajectories with tangent $\bar{v}^{a}$. In the averaging approach, it makes more sense to replace "individual galaxies" with the averaging domains considered as physically infinitesimal cells - the "points" of the averaged manifold $\overline{\mathcal{M}}$. This is physically reasonable since we know after all, that individual galaxies exhibit peculiar motions, undergo mergers and so on. This idea is also more in keeping with the notion that the Universe is homogeneous and isotropic only on the largest scales, which are much larger than the scale of individual galaxies. (See also Ref. [10] for a discussion of how this assumption of treating the averaging domains as being effectively point-like, is essential for the idempotency of the averaging operation.)

Consider any $3+1$ spacetime splitting in the form of a lapse function $N\left(t, x^{J}\right)$, a shift vector $N^{A}\left(t, x^{J}\right)$, and a metric for the 3 -geometry $h_{A B}\left(t, x^{J}\right)$, so that the line element on $\mathcal{M}$ can be written as

${ }^{(\mathcal{M})} d s^{2}=-\left(N^{2}-N_{A} N^{A}\right) d t^{2}+2 N_{B} d x^{B} d t+h_{A B} d x^{A} d x^{B}$,

where $N_{A}=h_{A B} N^{B}$. At first sight, it might seem reasonable to leave the choice of gauge arbitrary. One could then formally consider a coordination bivector given by the Eqns. (15) and (17), with $x^{i}$ denoting the coordinates in the chosen gauge and $\phi^{m}$ the VPCs; and demand for example, that the metric (47) (with say $N^{A}=0$ ) average out to the FLRW form (with a nonsynchronous time coordinate in general). This would imply

$$
\begin{aligned}
G_{00}=\left\langle\widetilde{g}_{00}\right\rangle_{S T} & =-f^{2}(t) ; G_{0 A}=\left\langle\widetilde{g}_{0 A}\right\rangle_{S T}=0 ; \\
G_{A B} & =\left\langle\widetilde{g}_{A B}\right\rangle_{S T}=a^{2}(t) \delta_{A B} .
\end{aligned}
$$

There is no a priori reason to assume that the functions $f(t)$ and $a(t)$ are related. Note that the condition on the bilocal extension $\widetilde{g}_{0 A}\left(x^{\prime}, x\right)$ is in general nontrivial even when the components $g_{0 A}(x)$ are chosen to be zero. In the Appendix A 1 we show that with the above assumptions, for a general lapse function $N$, the conditions $\mathbf{D}_{\bar{\Omega}} \bar{g}^{a b}=0$ (Eqn. (31)) also allow us to choose

$$
U^{i j} \equiv \bar{g}^{i j}-G^{i j}=0 .
$$

However, it turns out that for a general lapse function $N$, the explicit form of the correlation terms in the averaged equations with the assumptions (48), is rather complicated (although one can write down these terms formally in a relatively compact manner). On the other hand, if we make the assumption that the spatial sections on $\mathcal{M}$ leading to the spatial metric (42) on $\overline{\mathcal{M}}$, are spatial sections in a volume preserving gauge, then the correlation terms simplify greatly. This is not surprising since the MG formalism is nicely adapted to the choice of volume preserving coordinates.

We therefore adopt the following procedure : We will first make the set of assumptions which allow us to use a volume preserving gauge. Using these assumptions in the 
remainder of this section, we will introduce the notion of spatial averaging within the MG framework. In Section IV we will calculate the correlation terms and display the modified equations resulting from this particular choice of gauge. This exercise can be thought of as a toy calculation with simplifying assumptions. Following that calculation we will show how the correlation terms can be generalized to the more physically relevant case where the gauge in the inhomogeneous metric is (formally) left unspecified.

To begin our first calculation, we perform a coordinate transformation and shift to the gauge wherein the new lapse function $N$ is given by $N=1 / \sqrt{h}$ where $h$ is the determinant of the new 3-metric $h_{A B}$. In general, one will now be left with a non-zero shift vector $N^{A}$; however, the condition $N \sqrt{h}=1$ ensures that the coordinates we are now using are volume preserving, since the metric determinant is given by $g=-N^{2} h=-1=$ constant. We denote these volume preserving coordinates (VPCs) by $(\bar{t}, \mathbf{x})=\left(\bar{t}, x^{A}\right)=(\bar{t}, x, y, z)$, and will assume that the spatial coordinates are non-compact. For simplicity, we make the added assumption that $N^{A}=0$ in the inhomogenous geometry [15], so that $g_{\bar{t} \bar{t}}=-N^{2}=-1 / h$ and $g_{\bar{t} A}=0$. The line element for the inhomogenous manifold $\mathcal{M}$ becomes

$$
{ }^{(\mathcal{M})} d s^{2}=-\frac{d \bar{t}^{2}}{h(\bar{t}, \mathbf{x})}+h_{A B}(\bar{t}, \mathbf{x}) d x^{A} d x^{B} .
$$

Note that in this gauge, the average takes on a particularly simple form : for a tensor $p_{j}^{i}(x)$, with a spacetime averaging domain given by the "cuboid" $\boldsymbol{\Sigma}$ defined by

$\boldsymbol{\Sigma}=\{(\bar{t}, x, y, z) \mid-T / 2<\bar{t}<T / 2,-L / 2<x, y, z<L / 2\}$,

where $T$ and $L$ are averaging time and length scales respectively, the average is given by

$$
\begin{aligned}
& \left\langle\widetilde{p}_{j}^{i}\right\rangle_{S T}(\bar{t}, \mathbf{x})=\left\langle p_{j}^{i}\right\rangle_{S T}(\bar{t}, \mathbf{x}) \\
& =\frac{1}{T L^{3}} \int_{\bar{t}-T / 2}^{\bar{t}+T / 2} d t^{\prime} \int_{-L / 2}^{+L / 2} d x^{\prime} d y^{\prime} d z^{\prime}\left[p_{j}^{i}\left(t^{\prime}, x^{\prime}, y^{\prime}, z^{\prime}\right)\right],
\end{aligned}
$$

where the limits on the spatial integral are understood to hold for all three spatial coordinates. We define the "spatial averaging limit" as the limit $T \rightarrow 0$ (or $T \ll$ $\left.L_{\text {Hubble }}\right)$ which is interpreted as providing a definition of the average on a spatial domain corresponding to a "thin" time slice, the averaging operation now being given by

$$
\begin{aligned}
& \left\langle p_{j}^{i}\right\rangle(\bar{t}, \mathbf{x}) \\
& =\frac{1}{L^{3}} \int_{-L / 2}^{+L / 2} d x^{\prime} d y^{\prime} d z^{\prime}\left[p_{j}^{i}\left(\bar{t}, x^{\prime}, y^{\prime}, z^{\prime}\right)\right]+\mathcal{O}\left(T L_{\text {Hubble }}^{-1}\right) .
\end{aligned}
$$

(Note the time dependence of the integrand.) Henceforth, averaging will refer to spatial averaging, and will be denoted by $\langle\ldots\rangle$, in contrast to the spacetime averaging considered thus far (denoted by $\langle\ldots\rangle_{S T}$ ). The choice of a cube with sides of length $L$ as the spatial averaging domain was arbitrary, and is in fact not essential for any of the calculations to follow. In particular, all calculations can be performed with a spatial domain of arbitrary shape [16]. We will only use the cube for definiteness and simplicity in displaying equations. (An assumption essentially amounting to the limit in Eqn. (53) was also made in Ref. [13], in the context of averaging the spherically symmetric and inhomogeneous LemaîtreTolman-Bondi models.) The significance of introducing a spatial averaging in this manner is that the construction of spatial averaging is not isolated from spacetime averaging, but is a special limiting case of the latter and is, in fact, still a fully covariant operation.

For the volume preserving gauge, we now make the averaging assumption (48) (with the averaging scale $L=$ $L_{\text {FLRW }}$ ), which reduces to

$$
\begin{gathered}
G_{\bar{t} \bar{t}}=\left\langle g_{\bar{t} \bar{t}}\right\rangle=\left\langle\frac{-1}{h}\right\rangle=-f^{2}(\bar{t}) ; \\
G_{A B}=\left\langle h_{A B}\right\rangle=\bar{a}^{2}(\bar{t}) \delta_{A B},
\end{gathered}
$$

where $\bar{a}$ and $f$ are some functions of the time coordinate alone. A few remarks are in order on this particular choice of assumptions. Apart from the fact that the spacetime averaging operation takes on its simplest possible form (52) in this gauge and allows a transparent definition of the spatial averaging limit, it can also be shown that the assumptions in Eqn. (54) are sufficient to establish the following relations :

$$
f^{2}(\bar{t})=\left\langle\frac{1}{h}\right\rangle=\frac{1}{\langle h\rangle}=\frac{1}{\bar{a}^{6}} .
$$

Here the second equality arises from the condition $\bar{g}^{i j}=$ $G^{i j}$ which can be assumed whenever the averaged metric is of the FLRW form (see Appendix (A 1)). The last equality follows on considering the conditions $\left\langle\widetilde{\Gamma}_{b c}^{a}\right\rangle=$ ${ }^{(\mathrm{FLRW})} \Gamma_{b c}^{a}$ in obvious notation, (the basic assumption of the MG averaging scheme), details of which can be found in the Appendix (A2). Eqn. (55) reduces the line element on $\overline{\mathcal{M}}$ to the form

$$
{ }^{(\overline{\mathcal{M}})} d s^{2}=-\frac{d \bar{t}^{2}}{\bar{a}^{6}(\bar{t})}+\bar{a}^{2}(\bar{t}) \delta_{A B} d x^{A} d x^{B} .
$$

The line element in Eqn. (56) clearly corresponds to the FLRW metric in a volume preserving gauge. In other words, the (spatial) average of the inhomogeneous geometry in the volume preserving gauge leads to a geometry with homogeneous and isotropic spatial sections, also in a volume preserving gauge. Note that the gauge in Eqn. (56) ) for the FLRW spacetime differs from the standard synchronous and comoving gauge, only by a redefinition of the time coordinate. The vector field $\bar{v}^{a}$ introduced at the beginning of this section and which defines the FLRW spatial sections, is now given by

$$
\bar{v}^{a}=\left(\bar{a}^{3}, 0,0,0\right) \quad ; \quad \bar{v}_{a}=G_{a b} \bar{v}^{b}=\left(-\frac{1}{\bar{a}^{3}}, 0,0,0,\right) .
$$


Before proceeding to the calculation of the correlation terms and the averaged Einstein equations, we briefly describe why it is important to consider the spatial averaging limit of the MG averaging operation. The key idea to emphasize is that an average of the homogeneous and isotropic FLRW geometry, should give back the same geometry. Since the FLRW geometry has a preferred set of spatial sections, it is important therefore to perform the averaging over these sections. Further, since the FLRW metric adapted to its preferred spatial sections depends on the time coordinate, it is also essential that the spacetime average should involve a time range that is short compared to the scale over which say the scale factor changes significantly. (See also Sec. 4 of Ref. 10.) Clearly then, averaging the FLRW metric (denoted ${ }^{(F L R W)} g_{a b}$ ) given in Eqn. (56) (which is in volume preserving gauge) will strictly yield the same metric only in the limit $T \rightarrow 0$. Namely, for the cuboid $\boldsymbol{\Sigma}$ defined in Eqn. (51)

$$
\begin{aligned}
\left\langle(F L R W) \widetilde{g}_{a b}\right\rangle & =\lim _{T \rightarrow 0} \frac{1}{T L^{3}} \int_{\Sigma} d t^{\prime} d^{3} x^{(F L R W)} g_{a b}\left(t^{\prime}, \mathbf{x}^{\prime}\right) \\
& ={ }^{(F L R W)} g_{a b}
\end{aligned}
$$

which should be clear from the definition of the metric. The result $\left\langle(F L R W) \widetilde{g}_{a b}\right\rangle={ }^{(F L R W)} g_{a b}$ in the spatial averaging limit can also be shown to hold for the FLRW metric in synchronous gauge, where the coordination bivector $\mathcal{W}_{j}^{a^{\prime}}$ can be easily computed using the transformation from the $\operatorname{VPCs}\left(\bar{t}, x^{A}\right)$ to the synchronous coordinates $\left(\tau, y^{A}\right)$ given by

$$
\tau=\int^{\bar{t}} \frac{d t}{\bar{a}^{3}(t)} \quad ; \quad y^{A}=x^{A} .
$$

The transformation (59) will also later allow us to write the averaged equations in the synchronous gauge for the averaged geometry.

We now proceed to calculating the correlation 2-form $\mathbf{Z}^{a{ }^{i} j}$ and thereby the averaged Einstein equations.

\section{THE CORRELATION 2-FORM AND THE AVERAGED FIELD EQUATIONS}

\section{A. Results for the Volume Preserving Gauge}

We start by defining (in any gauge with $N^{A}=0$ ) the expansion tensor $\Theta_{B}^{A}$ by

$$
\Theta_{B}^{A} \equiv \frac{1}{2 N} h^{A C} \dot{h}_{C B},
$$

where the dot will always refer to a derivative with respect to the VPC time $\bar{t}$, and $h^{A B}$ is the inverse of the 3 -metric $h_{A B}$. (This also gives the symmetric tensor $\Theta_{A B}=(1 / 2 N) \dot{h}_{A B}$, which is the negative of the extrinsic curvature tensor.) The traceless symmetric shear tensor $\sigma_{B}^{A}$ and the shear scalar $\sigma^{2}$ are defined by

$$
\sigma_{B}^{A} \equiv \Theta_{B}^{A}-(\Theta / 3) \delta_{B}^{A} ; \quad \sigma^{2} \equiv \frac{1}{2} \sigma_{B}^{A} \sigma_{A}^{B}
$$

where $\Theta \equiv \Theta_{A}^{A}=(1 / N) \partial_{\bar{t}} \ln \sqrt{h}$ is the expansion scalar.

The connection 1-forms $\boldsymbol{\omega}^{i}{ }_{j}=\Gamma_{j k}^{i} \mathbf{d} x^{k}$ can be easily calculated in terms of the expansion tensor, for an arbitrary lapse function $N$. Specializing to the volume preserving gauge $\left(N=h^{-1 / 2}\right)$, the bilocal extensions $\Omega^{i}{ }_{j}$ of the connection 1 -forms are trivial and are simply given by

$$
\boldsymbol{\Omega}_{j}^{i}\left(x^{\prime}, x\right)=\Gamma_{j k}^{i}\left(x^{\prime}\right) \mathbf{d} x^{k} .
$$

Since $G_{a b}=\bar{g}_{a b}$, the connection 1-forms $\overline{\boldsymbol{\Omega}}^{i}{ }_{j}$ for the averaged manifold $\overline{\mathcal{M}}$ are constructed using the FLRW metric in volume preserving gauge given in Eqn. (56), and can also be easily evaluated.

We can now construct the correlation 2 -form $\mathbf{Z}_{b j}^{a}{ }_{b j}$ defined in Eqn. (22). For completeness, we will display all the nontrivial components $\mathbf{Z}_{b j}^{a}{ }_{b j}$, although not all of them will be relevant for the final equations. The condition $N=h^{-1 / 2}$ has the effect that several of the Christoffel symbols become related to each other. For example, we have $\Gamma_{00}^{0}=-\partial_{\bar{t}}(\ln \sqrt{h})=-\Gamma_{0 A}^{A}=-(1 / \sqrt{h}) \Theta$, and so on. In the following, ${ }^{(3)} \Gamma_{B C}^{A}$ denotes the Christoffel symbol built from the 3-metric $h_{A B}$, and we have defined

$$
H \equiv \frac{1}{\bar{a}} \frac{d \bar{a}}{d \bar{t}}
$$


We have,

$$
\begin{aligned}
& \mathbf{Z}^{0}{ }_{0}^{0}{ }_{A}=-\mathbf{Z}^{0}{ }_{A 0}^{0} \\
& =\left[\left\langle\Theta \Theta_{A J}\right\rangle+\left\langle\partial_{A}(\ln \sqrt{h}) \partial_{J}(\ln \sqrt{h})\right\rangle-3 \bar{a}^{8} H^{2} \delta_{A J}\right] \mathbf{d} x^{J} \wedge \mathbf{d} \bar{t}+\left\langle\sqrt{h} \Theta_{A J} \partial_{K}(\ln \sqrt{h})\right\rangle \mathbf{d} x^{J} \wedge \mathbf{d} x^{K}, \\
& \mathbf{Z}_{0 \quad{ }_{0}{ }_{0}}=-\mathbf{Z}^{A}{ }_{0}^{0} 0 \\
& =\left[\left\langle\frac{1}{h} \Theta \Theta_{J}^{A}\right\rangle+\left\langle\frac{1}{h} h^{A K} \partial_{K}(\ln \sqrt{h}) \partial_{J}(\ln \sqrt{h})\right\rangle-3 H^{2} \delta_{J}^{A}\right] \mathbf{d} x^{J} \wedge \mathbf{d} \bar{t}+\left\langle\frac{1}{\sqrt{h}} \Theta_{J}^{A} \partial_{K}(\ln \sqrt{h})\right\rangle \mathbf{d} x^{J} \wedge \mathbf{d} x^{K}, \\
& \mathbf{Z}_{0 B}^{0}{ }_{0}^{A}=-\mathbf{Z}_{B}^{A}{ }_{B}^{0} \\
& =\left[\left\langle\frac{1}{\sqrt{h}} \Theta^{(3)} \Gamma_{B J}^{A}\right\rangle-\left\langle\frac{1}{\sqrt{h}} \Theta_{B}^{A} \partial_{J}(\ln \sqrt{h})\right\rangle\right] \mathbf{d} x^{J} \wedge \mathbf{d} \bar{t}+\left\langle{ }^{(3)} \Gamma_{B J}^{A} \partial_{K}(\ln \sqrt{h})\right\rangle \mathbf{d} x^{J} \wedge \mathbf{d} x^{K}, \\
& \mathbf{Z}^{0}{ }_{A B}{ }_{B}=\left[2\left\langle\sqrt{h} \partial_{[A}(\ln \sqrt{h}) \Theta_{B] J}\right\rangle\right] \mathbf{d} x^{J} \wedge \mathbf{d} \bar{t}+\left[\left\langle h \Theta_{A J} \Theta_{B K}\right\rangle-\bar{a}^{16} H^{2} \delta_{A J} \delta_{B K}\right] \mathbf{d} x^{J} \wedge \mathbf{d} x^{K}, \\
& \mathbf{Z}^{0}{ }_{A}{ }_{0}{ }_{0}=-\mathbf{Z}^{B}{ }_{0}^{0}{ }_{A} \\
& =\left[\left\langle\frac{1}{\sqrt{h}} \partial_{A}(\ln \sqrt{h}) \Theta_{J}^{B}\right\rangle-\left\langle\frac{1}{\sqrt{h}} h^{B K} \partial_{K}(\ln \sqrt{h}) \Theta_{A J}\right\rangle\right] \mathbf{d} x^{J} \wedge \mathbf{d} \bar{t}+\left[\left\langle\Theta_{A J} \Theta_{K}^{B}\right\rangle-\bar{a}^{8} H^{2} \delta_{A J} \delta_{K}^{B}\right] \mathbf{d} x^{J} \wedge \mathbf{d} x^{K}, \\
& \mathbf{Z}^{0}{ }_{A}{ }^{B}{ }_{C}=-\mathbf{Z}^{B}{ }_{C}{ }^{0}{ }_{A} \\
& =\left[\left\langle\partial_{A}(\ln \sqrt{h})^{(3)} \Gamma_{C J}^{B}\right\rangle+\left\langle\Theta_{A J} \Theta_{C}^{B}\right\rangle-\bar{a}^{8} H^{2} \delta_{A J} \delta_{C}^{B}\right] \mathbf{d} x^{J} \wedge \mathbf{d} \bar{t}+\left\langle\sqrt{h} \Theta_{A J}{ }^{(3)} \Gamma_{C K}^{B}\right\rangle \mathbf{d} x^{J} \wedge \mathbf{d} x^{K}, \\
& \mathbf{Z}_{0}^{A B}{ }_{0}=\left[2\left\langle\frac{1}{h^{3 / 2}} \partial_{K}(\ln \sqrt{h}) h^{K[A} \Theta_{J}^{B]}\right\rangle\right] \mathbf{d} x^{J} \wedge \mathbf{d} \bar{t}+\left[\left\langle\frac{1}{h} \Theta_{J}^{A} \Theta_{K}^{B}\right\rangle-H^{2} \delta_{J}^{A} \delta_{K}^{B}\right] \mathbf{d} x^{J} \wedge \mathbf{d} x^{K}, \\
& \mathbf{Z}^{A B}{ }_{0}{ }_{C}=-\mathbf{Z}^{B}{ }_{C}{ }_{0}{ }_{0} \\
& =\left[\left\langle\frac{1}{h} h^{A K} \partial_{K}(\ln \sqrt{h})^{(3)} \Gamma_{C J}^{B}\right\rangle+\left\langle\frac{1}{h} \Theta_{J}^{A} \Theta_{C}^{B}\right\rangle-H^{2} \delta_{J}^{A} \delta_{C}^{B}\right] \mathbf{d} x^{J} \wedge \mathbf{d} \bar{t}+\left\langle\frac{1}{\sqrt{h}} \Theta_{J}^{A}{ }^{(3)} \Gamma_{C K}^{B}\right\rangle \mathbf{d} x^{J} \wedge \mathbf{d} x^{K}, \\
& \mathbf{Z}^{A}{ }_{B}{ }^{C}{ }_{D}=\left[\left\langle\frac{1}{\sqrt{h}} \Theta_{D}^{C}{ }^{(3)} \Gamma_{B J}^{A}\right\rangle-\left\langle\frac{1}{\sqrt{h}} \Theta_{B}^{A}{ }^{(3)} \Gamma_{D J}^{C}\right\rangle\right] \mathbf{d} x^{J} \wedge \mathbf{d} \bar{t}+\left\langle{ }^{(3)} \Gamma_{B J}^{A}{ }^{(3)} \Gamma_{D K}^{C}\right\rangle \mathbf{d} x^{J} \wedge \mathbf{d} x^{K}
\end{aligned}
$$

where we have used the relation ${ }^{(3)} \Gamma_{B J}^{J}=\partial_{B}(\ln \sqrt{h})$, and the square brackets around indices indicate antisymmetrization $\left(p_{[i j]}=(1 / 2 !)\left(p_{i j}-p_{j i}\right)\right.$. $)$

It is now straightforward to use the relations in Eqn. (36) (note the unconventional normalization of the 2form) to read off the components $Z_{b m}^{a}{ }_{b n}^{i}$ and hence perform the required summations to construct $Z^{a}{ }_{i j b}$. This, together with the relation $\bar{g}^{a b}=G^{a b}$, allows us to construct the correlation tensor $C_{b}^{a}$ defined in Eqn. (37)

$$
C_{b}^{a}=\left(Z^{a}{ }_{i j b}-\frac{1}{2} \delta_{b}^{a} Z^{m}{ }_{i j m}\right) G^{i j} .
$$

Now, the components of the Einstein tensor $E_{b}^{a}$ for the averaged spacetime with metric (56) are given by

$$
\begin{aligned}
E_{\bar{t}}^{\bar{t}} & =3 \bar{a}^{6} H^{2} \quad ; \quad E_{A}^{\bar{t}}=0=E_{\bar{t}}^{B}, \\
E_{B}^{A} & =\bar{a}^{6} \delta_{B}^{A}\left[2\left(\frac{\ddot{\bar{a}}}{\bar{a}}+3 H^{2}\right)+H^{2}\right],
\end{aligned}
$$

where the peculiar splitting of terms in the last equation is for later convenience. Recall that the overdot denotes a derivative with respect to the VPC time $\bar{t}$, not synchronous time. In terms of the coordinate independent 
objects introduced in Eqn. (46), we have

$$
j_{1}(x)=-3 \bar{a}^{6} H^{2} \quad ; \quad j_{2}(x)=\bar{a}^{6}\left[2\left(\frac{\ddot{\bar{a}}}{\bar{a}}+3 H^{2}\right)+H^{2}\right] .
$$

¿From the averaged Einstein equations in (39) we next construct the scalar equations which in the standard case would correspond to the Friedmann equation and the Raychaudhuri equation. These correspond to the Einstein tensor components,

$$
E_{b}^{a} \bar{v}^{b} \bar{v}_{a}=j_{1}(x) ; \pi_{a}^{b} E_{b}^{a}+E_{b}^{a} \bar{v}^{b} \bar{v}_{a}=3 j_{2}(x)+j_{1}(x),
$$

and are given by

$$
\begin{aligned}
& 3 \bar{a}^{6} H^{2}=\left(\kappa T_{b}^{a}-C_{b}^{a}\right) \bar{v}_{a} \bar{v}^{b} \\
&=\kappa \bar{\rho}-\frac{1}{2}\left[\mathcal{Q}^{(1)}+\mathcal{S}^{(1)}\right], \\
& 6 \bar{a}^{6}\left(\frac{\ddot{\bar{a}}}{\bar{a}}+3 H^{2}\right)=\left(-\kappa T_{b}^{a}+C_{b}^{a}\right)\left(\bar{v}_{a} \bar{v}^{b}+\pi_{a}^{b}\right) \\
& \quad=-\kappa(\bar{\rho}+3 \bar{p})+2\left[\mathcal{Q}^{(1)}+\mathcal{Q}^{(2)}+\mathcal{S}^{(2)}\right] .
\end{aligned}
$$

Here Eqn. (69a) is the modified Friedmann equation and Eqn. (69b) the modified Raychaudhuri equation (in the volume preserving gauge on $\overline{\mathcal{M}}$ ). We have used Eqn. (45), with the overbar on $\rho$ and $p$ reminding us that they are expressed in terms of the nonsynchronous time $\bar{t}$, and we have defined the correlation terms

$$
\begin{gathered}
\mathcal{Q}^{(1)}=\bar{a}^{6}\left[\frac{2}{3}\left(\left\langle\frac{1}{h} \Theta^{2}\right\rangle-\frac{1}{\bar{a}^{6}}\left({ }^{\mathrm{F}} \Theta^{2}\right)\right)-2\left\langle\frac{1}{h} \sigma^{2}\right\rangle\right] \\
\frac{1}{\bar{a}^{6}}\left({ }^{\mathrm{F}} \Theta^{2}\right)=(3 H)^{2}, \\
\mathcal{S}^{(1)}=\frac{1}{\bar{a}^{2}} \delta^{A B}\left[\left\langle{ }^{(3)} \Gamma_{A C}^{J}{ }^{(3)} \Gamma_{B J}^{C}\right\rangle\right. \\
\left.-\left\langle\partial_{A}(\ln \sqrt{h}) \partial_{B}(\ln \sqrt{h})\right\rangle\right] \\
\mathcal{Q}^{(2)}=\bar{a}^{6}\left\langle\frac{1}{h} \Theta_{B}^{A} \Theta_{A}^{B}\right\rangle-\frac{1}{\bar{a}^{2}} \delta^{A B}\left\langle\Theta_{A J} \Theta_{B}^{J}\right\rangle \\
\mathcal{S}^{(2)}=\bar{a}^{6}\left\langle\frac{1}{h} h^{A B} \partial_{A}(\ln \sqrt{h}) \partial_{B}(\ln \sqrt{h})\right\rangle \\
-\frac{1}{\bar{a}^{2}} \delta^{A B}\left\langle\partial_{A}(\ln \sqrt{h}) \partial_{B}(\ln \sqrt{h})\right\rangle
\end{gathered}
$$

In defining $\mathcal{Q}^{(1)}$ we have used the relation $\Theta^{2}-\Theta_{B}^{A} \Theta_{A}^{B}=$ $(2 / 3) \Theta^{2}-2 \sigma^{2}$. $\mathcal{Q}^{(1)}$ and $\mathcal{Q}^{(2)}$ are correlations of the extrinsic curvature, whereas $\mathcal{S}^{(1)}$ and $\mathcal{S}^{(2)}$ are correlations restricted to the intrinsic 3-geometry of the spatial slices of $\mathcal{M}$. Since the components of $C_{b}^{a}$ are not explicitly constrained by Eqns. (27) and (28), we can treat the combinations $(1 / 2)\left(\mathcal{Q}^{(1)}+\mathcal{S}^{(1)}\right)=-C_{0}^{0}$ and
$2\left(\mathcal{Q}^{(1)}+\mathcal{Q}^{(2)}+\mathcal{S}^{(2)}\right)=\left(C_{A}^{A}-C_{0}^{0}\right)$ as independent, subject only to the differential constraints (41) which we will come to below.

As discussed in the beginning of Section [II] the remaining components of $C_{b}^{a}$ must be set to zero, giving constraints on the underlying inhomogeneous geometry. In coordinate independent language, these constraints read

$$
\begin{aligned}
& \pi_{k}^{b} C_{b}^{a} \bar{v}_{a}=0=\pi_{a}^{k} C_{b}^{a} \bar{v}^{b} ; \\
& \pi_{a}^{i} \pi_{k}^{b} C_{b}^{a}-\frac{1}{3} \pi_{k}^{i}\left(\pi_{a}^{b} C_{b}^{a}\right)=0 .
\end{aligned}
$$

Eqns. (71) reduce to the following for our specific choice of volume preserving coordinates,

$$
C_{A}^{0}=0 ; C_{0}^{A}=0 ; C_{B}^{A}-\frac{1}{3} \delta_{B}^{A}\left(C_{J}^{J}\right)=0,
$$

It can be shown that the VPC assumption $N=h^{-1 / 2}$ reduces the correlations $\mathcal{Q}^{(2)}$ and $\mathcal{S}^{(2)}$ defined in Eqns. (70c) and (70d), as well as several terms in the explicit expansion of Eqn. (72), to the form

$$
\frac{1}{\left\langle g_{00}\right\rangle}\left\langle g_{00} g^{A B} \Gamma_{b_{1} c_{1}}^{a_{1}} \Gamma_{j_{1} k_{1}}^{i_{1}}\right\rangle-\left\langle g^{A B}\right\rangle\left\langle\Gamma_{b_{2} c_{2}}^{a_{2}} \Gamma_{j_{2} k_{2}}^{i_{2}}\right\rangle \text {. }
$$

The assumption in Eqn. (30b) (which is fundamental to the MG formalism) now shows that one can write

$$
\begin{aligned}
\left\langle g_{00} g^{A B} \Gamma_{b c}^{a} \Gamma_{j k}^{i}\right\rangle & =\left\langle g_{00} g^{A B}\right\rangle\left\langle\Gamma_{b c}^{a} \Gamma_{j k}^{i}\right\rangle \\
& =-\left\langle\frac{h^{A B}}{h}\right\rangle\left\langle\Gamma_{b c}^{a} \Gamma_{j k}^{i}\right\rangle .
\end{aligned}
$$

An interesting point is that the VPC assumption $N=h^{-1 / 2}$ further allows us to assume $\left\langle h^{A B} / h\right\rangle=$ $\left\langle h^{A B}\right\rangle\langle 1 / h\rangle$ consistently with the formalism (details in Appendix (A 2) ). Using Eqn. (55) this gives us

$$
\left\langle\frac{h^{A B}}{h}\right\rangle=\frac{1}{\bar{a}^{6}}\left\langle h^{A B}\right\rangle \text {. }
$$

This shows that the correlation terms $\mathcal{Q}^{(2)}$ and $\mathcal{S}^{(2)}$ in fact vanish,

$$
\mathcal{Q}^{(2)}=0=\mathcal{S}^{(2)},
$$

and leads to some remarkable cancellations in Eqns. (72), which simplify to give

$$
\begin{gathered}
\delta^{J K}\left[\left\langle\sqrt{h} \Theta_{J B}{ }^{(3)} \Gamma_{A K}^{B}\right\rangle-\left\langle\sqrt{h} \Theta_{J K}{ }^{(3)} \Gamma_{A B}^{B}\right\rangle\right]=0 \\
\delta^{J K}\left\langle\frac{1}{\sqrt{h}} \Theta_{K}^{B}{ }^{(3)} \Gamma_{J B}^{A}\right\rangle-\delta^{A J}\left\langle\frac{1}{\sqrt{h}} \Theta_{K}^{K}{ }^{(3)} \Gamma_{J B}^{B}\right\rangle=0 \\
\delta^{J K}\left\langle{ }^{(3)} \Gamma_{J C}^{A}{ }^{(3)} \Gamma_{K B}^{C}\right\rangle-\delta^{A J}\left\langle{ }^{(3)} \Gamma_{J C}^{C}{ }^{(3)} \Gamma_{B K}^{K}\right\rangle \\
=\frac{1}{3} \delta_{B}^{A}\left(\bar{a}^{2} \mathcal{S}^{(1)}\right)
\end{gathered}
$$


These simplifications are solely a consequence of assuming that the inhomogeneous metric in the volume preserving gauge averages out to give the FLRW metric in standard form. In general, these simplifications will not occur when the standard FLRW metric arises from an arbitrary choice of gauge for the inhomogeneous metric.

In order to come as close as possible to the standard approach in Cosmology, we will now rewrite the scalar equations (69) (which are the cosmologically relevant ones) after performing the transformation given in Eqn. (59) in order to get the FLRW metric to the form

$$
{ }^{(\overline{\mathcal{M}})} d s^{2}=-d \tau^{2}+a^{2}(\tau) \delta_{A B} d y^{A} d y^{B} \quad ; \quad a(\tau)=\bar{a}(\bar{t}(\tau)) .
$$

Since Eqns. (69) are scalar equations, this transformation only has the effect of reexpressing all the terms as functions of the synchronous time $\tau$. Although the transformation will change the explicit form of the coordination bivector $\mathcal{W}_{j}^{a^{\prime}}$, this change involves only the time coordinate, and in the spatial averaging limit there is no difference between averages computed in the VPCs and those computed after the time redefinition. This again emphasizes the importance of the spatial averaging limit of spacetime averaging, if we are to succeed operationally in explicitly displaying the correlations as corrections to the standard cosmological equations. The correlation terms in Eqns. (70) are therefore still interpreted with respect to the volume preserving gauge, but are treated as functions of $\tau$. For the scale factor on the other hand, we have

$$
\bar{a}^{3} H=\frac{1}{a} \frac{d a}{d \tau} \equiv H_{\mathrm{FLRW}} \quad ; \quad \bar{a}^{6}\left(\frac{\ddot{\bar{a}}}{\bar{a}}+3 H^{2}\right)=\frac{1}{a} \frac{d^{2} a}{d \tau^{2}} .
$$

Further writing

$$
\rho(\tau)=\bar{\rho}(\bar{t}(\tau)) \quad ; \quad p(\tau)=\bar{p}(\bar{t}(\tau)),
$$

equations (69) become

$$
\begin{aligned}
H_{\mathrm{FLRW}}^{2} & =\frac{8 \pi G_{N}}{3} \rho-\frac{1}{6}\left[\mathcal{Q}^{(1)}+\mathcal{S}^{(1)}\right], \\
\frac{1}{a} \frac{d^{2} a}{d \tau^{2}} & =-\frac{4 \pi G_{N}}{3}(\rho+3 p)+\frac{1}{3} \mathcal{Q}^{(1)} .
\end{aligned}
$$

We emphasize that the quantities $\mathcal{Q}^{(1)}$ and $\mathcal{S}^{(1)}$, defined in Eqns. (70a and (70b as correlations in the volume preserving gauge, are to be thought of as functions of the synchronous time $\tau$, where the coordinate $\tau$ itself was defined after the spatial averaging. Such an identification is justified since we are dealing with scalar combinations of these quantities. Note that $\mathcal{Q}^{(1)}$ and $\mathcal{S}^{(1)}$ can be treated independently, apart from the constraints imposed by Eqn. (41), which we turn to next. These conservation conditions can be decomposed into a scalar part and a 3-vector part, given respectively by

$$
\bar{v}^{b} C_{b ; a}^{a}=0 ; \pi_{k}^{b} C_{b ; a}^{a}=0
$$

In the synchronous gauge (78) for the FLRW metric, the scalar equation reads

$$
\left(\partial_{\tau} \mathcal{Q}^{(1)}+6 H_{\mathrm{FLRW}} \mathcal{Q}^{(1)}\right)+\left(\partial_{\tau} \mathcal{S}^{(1)}+2 H_{\mathrm{FLRW}} \mathcal{S}^{(1)}\right)=0 .
$$

We recall that this equation is a consequence of setting the correlation 3 -form and the correlation 4 -form to zero, and it relates the evolution of $\mathcal{Q}^{(1)}$ and $\mathcal{S}^{(1)}$. The 3 -vector equation (on imposing the first set of conditions in Eqn. (71) ) simply gives $\partial_{\tau} C_{A}^{\tau}=0$, so that $C_{A}^{\tau}=0=$ constant, which also implies that $C_{\tau}^{A}=0=$ constant and hence this equation gives nothing new. (We have used the relations $C_{0}^{0}=C_{\tau}^{\tau}, C_{A}^{0}=\bar{a}^{3} C_{A}^{\tau}$ and $C_{0}^{A}=\left(1 / \bar{a}^{3}\right) C_{\tau}^{A}$ where 0 denotes the nonsynchronous time coordinate $\bar{t}$.)

The cosmological equations (81), along with the constraint equations (77) and (83) are the key results of this section. Subject to the acceptance of the volume preserving gauge on the underlying manifold $\mathcal{M}$ they can in principle be used to study the role of the correction terms resulting from spatial averaging.

\section{B. Results for an arbitrary gauge choice}

In this subsection, we will display the results obtained on assuming that the metric

$$
{ }^{(\mathcal{M})} d s^{2}=-N^{2}(t, \mathbf{x}) d t^{2}+h_{A B}(t, \mathbf{x}) d x^{A} d x^{B},
$$

averages out to the FLRW metric in standard form with a nonsynchronous time coordinate $t$ in general, to give

$$
{ }^{(\overline{\mathcal{M}})} d s^{2}=-f^{2}(t) d t^{2}+\bar{a}^{2}(t) \delta_{A B} d x^{A} d x^{B} .
$$

In other words, we are assuming that the relations in Eqn. (48) hold. Note that the averaging operation is no longer trivial, although we are still assuming an averaging on domains corresponding to "thin" time slices. We again split the averaged Einstein equations into scalar equations, and 3-vector and traceless 3 -tensor equations. After transforming to the synchronous time coordinate $\tau$, now defined by

$$
\tau=\int^{t} f\left(t^{\prime}\right) d t^{\prime}
$$

and again defining $H \equiv(1 / \bar{a})(d \bar{a} / d t)$ and $H_{\text {FLRW }} \equiv$ $(1 / a)(d a / d \tau)$ with $a(\tau)=\bar{a}(t(\tau))$, the modified Friedmann and Raychaudhuri equations read

$$
\begin{aligned}
& H_{\mathrm{FLRW}}^{2}=\frac{8 \pi G_{N}}{3} \rho-\frac{1}{6}\left[\tilde{\mathcal{P}}^{(1)}+\tilde{\mathcal{S}}^{(1)}\right], \\
& \frac{1}{a} \frac{d^{2} a}{d \tau^{2}}=-\frac{4 \pi G_{N}}{3}(\rho+3 p)+\frac{1}{3}\left[\tilde{\mathcal{P}}^{(1)}+\tilde{\mathcal{P}}^{(2)}+\tilde{\mathcal{S}}^{(2)}\right],
\end{aligned}
$$


where the correlation terms are now defined using the relations,

$$
\begin{aligned}
& \tilde{\mathcal{P}}^{(1)}=\frac{1}{f^{2}}\left[\left\langle\widetilde{\Gamma}_{0 A}^{A} \widetilde{\Gamma}_{0 B}^{B}\right\rangle-\left\langle\widetilde{\Gamma}_{0 B}^{A} \widetilde{\Gamma}_{0 A}^{B}\right\rangle-6 H^{2}\right], \\
& \tilde{\mathcal{S}}^{(1)}=\left\langle\widetilde{g}^{J K}\right\rangle\left[\left\langle\widetilde{\Gamma}_{J B}^{A} \widetilde{\Gamma}_{K A}^{B}\right\rangle-\left\langle\widetilde{\Gamma}_{J A}^{A} \widetilde{\Gamma}_{K B}^{B}\right\rangle\right], \\
& \tilde{\mathcal{P}}^{(2)}+\tilde{\mathcal{P}}^{(1)}=-\frac{1}{f^{2}}\left\langle\widetilde{\Gamma}_{0 A}^{A} \widetilde{\Gamma}_{00}^{0}\right\rangle-\left\langle\widetilde{g}^{J K}\right\rangle\left\langle\widetilde{\Gamma}_{J A}^{0} \widetilde{\Gamma}_{0 K}^{A}\right\rangle \\
& +\frac{3 H}{f^{2}}\left(\partial_{t}(\ln f)+H\right) \text {, } \\
& \tilde{\mathcal{S}}^{(2)}=\frac{1}{f^{2}}\left\langle\widetilde{\Gamma}_{00}^{A} \widetilde{\Gamma}_{A 0}^{0}\right\rangle+\left\langle\widetilde{g}^{J K}\right\rangle\left\langle\widetilde{\Gamma}_{J 0}^{0} \widetilde{\Gamma}_{K A}^{A}\right\rangle
\end{aligned}
$$

We emphasize that averaging here refers to spatial averaging. Also $\left\langle\widetilde{g}^{J K}\right\rangle=G^{J K}=\left(1 / \bar{a}^{2}\right) \delta^{J K}$, and the index 0 refers to the nonsynchronous time $t$. It is easy to check that $\tilde{\mathcal{P}}^{(1)}$ and $\tilde{\mathcal{P}}^{(1)}+\tilde{\mathcal{P}}^{(2)}$ correspond to correlations of (the bilocal extensions of) the extrinsic curvature with itself and with the time derivative of the lapse function. $\tilde{\mathcal{S}}^{(1)}$ corresponds to correlations between the bilocal extensions of the Christoffel symbols of the 3 -geometry, and $\tilde{\mathcal{S}}^{(2)}$ to correlations of the extension of the spatial derivative of the lapse function with itself and with the Christoffel symbols of the 3-geometry. Due to the way we have defined these correlations, one can also check that when the lapse function satisfies $N \sqrt{h}=1$ (so that the averaging becomes trivial), we have $\tilde{\mathcal{P}}^{(1)}=\mathcal{Q}^{(1)}$, $\tilde{\mathcal{S}}^{(1)}=\mathcal{S}^{(1)}$, and $\tilde{\mathcal{P}}^{(2)}=0=\tilde{\mathcal{S}}^{(2)}$, where $\mathcal{Q}^{(1)}$ and $\mathcal{S}^{(1)}$ were defined in Eqns. (70). The 3 -vector and traceless 3-tensor equations become

$$
\begin{aligned}
\frac{1}{f^{2}}\left[\left\langle\widetilde{\Gamma}_{0 A}^{0} \widetilde{\Gamma}_{B 0}^{B}\right\rangle-\right. & \left.\left\langle\widetilde{\Gamma}_{0 B}^{0} \widetilde{\Gamma}_{A 0}^{B}\right\rangle\right] \\
& +\left\langle\widetilde{g}^{J K}\right\rangle\left[\left\langle\widetilde{\Gamma}_{J B}^{0} \widetilde{\Gamma}_{A K}^{B}\right\rangle-\left\langle\widetilde{\Gamma}_{J A}^{0} \widetilde{\Gamma}_{B K}^{B}\right\rangle\right]=0
\end{aligned}
$$

$$
\begin{aligned}
\frac{1}{f^{2}}\left[\left\langle\widetilde{\Gamma}_{00}^{A} \widetilde{\Gamma}_{B 0}^{B}\right\rangle-\right. & \left.\left\langle\widetilde{\Gamma}_{00}^{B} \widetilde{\Gamma}_{B 0}^{A}\right\rangle\right] \\
& +\left\langle\widetilde{g}^{J K}\right\rangle\left[\left\langle\widetilde{\Gamma}_{J B}^{A} \widetilde{\Gamma}_{0 K}^{B}\right\rangle-\left\langle\widetilde{\Gamma}_{J 0}^{A} \widetilde{\Gamma}_{B K}^{B}\right\rangle\right]=0
\end{aligned}
$$

$$
\begin{aligned}
\frac{1}{f^{2}} & {\left[\left\langle\widetilde{\Gamma}_{B 0}^{A} \widetilde{\Gamma}_{0 m}^{m}\right\rangle-\left\langle\widetilde{\Gamma}_{m 0}^{A} \widetilde{\Gamma}_{0 B}^{m}\right\rangle\right] } \\
& +\left\langle\widetilde{g}^{J K}\right\rangle\left[\left\langle\widetilde{\Gamma}_{J m}^{A} \widetilde{\Gamma}_{K B}^{m}\right\rangle-\left\langle\widetilde{\Gamma}_{J B}^{A} \widetilde{\Gamma}_{K m}^{m}\right\rangle\right] \\
& =-\frac{1}{3} \delta_{B}^{A}\left[\tilde{\mathcal{P}}^{(2)}+\tilde{\mathcal{S}}^{(2)}-\tilde{\mathcal{S}}^{(1)}-\frac{9 H}{f^{2}}\left(H+\frac{1}{3} \partial_{t}(\ln f)\right)\right]
\end{aligned}
$$

where the lower case index $m$ in the last equation runs over all spacetime indices $0,1,2,3$, with the index 0 referring to the nonsynchronous time $t$. It is easy to check that Eqns. (89) reduce to Eqns. (77) with the choice $N=h^{-1 / 2}$. The condition $C_{b ; a}^{a}=0$ has the scalar part,

$$
\begin{aligned}
\left(\partial_{\tau} \tilde{\mathcal{P}}^{(1)}+6 H_{\mathrm{FLRW}} \tilde{\mathcal{P}}^{(1)}\right) & +\left(\partial_{\tau} \tilde{\mathcal{S}}^{(1)}+2 H_{\mathrm{FLRW}} \tilde{\mathcal{S}}^{(1)}\right) \\
& +4 H_{\mathrm{FLRW}}\left(\tilde{\mathcal{P}}^{(2)}+\tilde{\mathcal{S}}^{(2)}\right)=0,
\end{aligned}
$$

while the 3-vector part, as before, gives nothing new and simply states $\partial_{\tau} C_{A}^{\tau}=0$.

We can now state the main result of our paper in a clear and unambiguous manner, as follows: Having assumed that the FLRW spatial sections arise as the average of some gauge choice with lapse function $N(t, \mathbf{x})$, spatial 3metric $h_{A B}(t, \mathbf{x})$ and shift vector $N^{A}$ set to zero for convenience, we can construct the scalar quantities $C_{b}^{a} \bar{v}^{b} \bar{v}_{a}$ and $\pi_{a}^{b} C_{b}^{a}+C_{b}^{a} \bar{v}^{b} \bar{v}_{a}$ which, in coordinates natural to the FLRW metric take the form,

$$
\begin{aligned}
& C_{b}^{a} \bar{v}^{b} \bar{v}_{a}=\frac{1}{2}\left[\tilde{\mathcal{P}}^{(1)}+\tilde{\mathcal{S}}^{(1)}\right] ; \\
& \pi_{a}^{b} C_{b}^{a}+C_{b}^{a} \bar{v}^{b} \bar{v}_{a}=2\left[\tilde{\mathcal{P}}^{(1)}+\tilde{\mathcal{P}}^{(2)}+\tilde{\mathcal{S}}^{(2)}\right],
\end{aligned}
$$

with the various quantities being defined in Eqns. (88). These scalars modify the usual cosmological equations as shown in Eqns. (87), and are themselves subject to the differential conditions (90). In addition, for consistency of our assumptions with the formalism, the underlying inhomogeneous metric is also subject to the conditions (89).

The combinations on the right hand sides of the relations (91) can clearly be treated independently, apart from the conditions (90). Further, since the correlation 2-form has 40 independent components $Z^{a}{ }_{b m}{ }^{i}{ }_{j n}$ after imposing all algebraic constraints, and since none of the four quantities $\tilde{\mathcal{P}}^{(1)}, \tilde{\mathcal{P}}^{(2)}, \tilde{\mathcal{S}}^{(1)}$ and $\tilde{\mathcal{S}}^{(2)}$ are trivially related by these constraints, one can always treat these four functions independently of each other, subject only to the constraint in Eqn. (90). Before proceeding, we wish to make two remarks concerning the possible behaviour of the correction terms. It was mentioned in Ref. [12] that assuming only spatial correlations, i.e. assuming that all components of the correlation 2-form $\mathbf{Z}_{b j}^{a}{ }_{b j}$ with at least one 0 index vanish, the corrections must be of the form of a spatial curvature term in the FLRW equations. We can confirm this statement, since the above assumption amounts to setting $\tilde{\mathcal{P}}^{(1)}, \tilde{\mathcal{P}}^{(2)}$ and $\tilde{\mathcal{S}}^{(2)}$ to zero, leaving only $\tilde{\mathcal{S}}^{(1)}$ which must then evolve as $\sim a^{-2}$ because of Eqn. (90). Further, the main result of the Ref. [12] was that assuming the averaged metric to be FLRW in the conformal gauge (which corresponds to assuming $f(t)=\bar{a}(t)$ in our case) and further assuming all components of the correlation 2 -form to be constant, the corrections must again be of the form of a spatial curvature term. Eqns. (88) show that this is indeed the case, 
and Eqn. (90) shows that in this case one must have $\tilde{\mathcal{P}}^{(1)}+\tilde{\mathcal{P}}^{(2)}+\tilde{\mathcal{S}}^{(2)}=0$, with no condition on the constant $a^{2} \tilde{\mathcal{S}}^{(1)}$, which is consistent with a curvature term in the modified FLRW equations (87). Clearly though, the allowed behaviour of the correction terms which is consistent with Eqn. (90), is more general than that of a spatial curvature term, and it is not yet clear how these corrections behave in the real Universe.

It is only fair to say that much of what we have described is already implicit in the work of Zalaletdinov and collaborators. What we have done here is to spell it out explicitly, emphasizing the relevance of spatial averaging for Cosmology. Further, the correction terms resulting from averaging have been displayed explicitly as scalars, which could be of help in applications and comparison with observations. A hitherto unappreciated fact which emerges is that even if some inhomogeneous geometry yields an FLRW Universe upon averaging over sufficiently large scales, this is not sufficient to guarantee consistency with the averaged Einstein equations. The consistency conditions (89) must be satisfied - this fact could have potential significance in restricting the class of allowed initial perturbations in the early Universe, and should be investigated further.

Another important goal of our paper is to attempt to compare the approaches of Zalaletdinov and Buchert, a topic to which we now turn.

\section{A comparison with the averaging formalism of Buchert}

The averaging formalism developed by Buchert is based exclusively on the manifold $\mathcal{M}$, and there is no analog of the averaged manifold $\overline{\mathcal{M}}$ in this scheme. Given an inhomogeneous metric on $\mathcal{M}$ one takes the trace of the Einstein equations in the inhomogeneous geometry, and carries out a spatial averaging of the inhomogeneous scalar equations.

We recall in brief Buchert's construction [3], by first writing down the averaged equations for the simplest case of pressureless and irrotational inhomogeneous dust. The metric can be written in synchronous and comoving gauge as

$$
d s^{2}=-d t^{2}+b_{A B}(\mathbf{x}, t) d x^{A} d x^{B} .
$$

The Einstein equations can be split 3] into a set of scalar equations and a set of vector and traceless tensor equations. The scalar equations are the Hamiltonian constraint (93a) and the evolution equation for $\Theta$ (93b),

$$
\begin{gathered}
\mathcal{R}+\frac{2}{3} \Theta^{2}-2 \sigma^{2}=16 \pi G \rho, \\
\mathcal{R}+\partial_{t} \Theta+\Theta^{2}=12 \pi G \rho,
\end{gathered}
$$

where $\mathcal{R}$ is the Ricci scalar of the 3 -dimensional hypersurface of constant $t, \Theta$ and $\sigma^{2}$ are the expansion scalar and the shear scalar defined earlier and $\rho$ is the inhomogeneous matter density of the dust. Note that all quantities in Eqns. (93) generically depend on both position $\mathbf{x}$ and time $t$. Eqns. (93a) and (93b) can be combined to give Raychaudhuri's equation

$$
\partial_{t} \Theta+\frac{1}{3} \Theta^{2}+2 \sigma^{2}+4 \pi G \rho=0 .
$$

The continuity equation $\partial_{t} \rho=-\Theta \rho$ which gives the evolution of $\rho$, is consistent with Eqns. (93a), (93b). Only scalar Einstein equations are considered, since the spatial average of a scalar quantity can be defined in a gauge covariant manner, within a given foliation of space-time. We return to this point below. For the space-time described by (92), the spatial average of a scalar $\Psi(\mathbf{x}, t)$ over a comoving domain $\mathcal{D}$ at time $t$ is defined by

$$
\langle\Psi\rangle_{\mathcal{D}}=\frac{1}{V_{\mathcal{D}}} \int_{\mathcal{D}} d^{3} x \sqrt{b} \Psi,
$$

where $b$ is the determinant of the 3-metric $b_{A B}$ and $V_{\mathcal{D}}$ is the volume of the comoving domain given by $V_{\mathcal{D}}=\int_{\mathcal{D}} d^{3} x \sqrt{b}$. Spatial averaging is, by definition, not generally covariant. Thus the choice of foliation is relevant, and should be motivated on physical grounds. In the context of cosmology, averaging over freely-falling observers is a natural choice, especially when one intends to compare the results with standard FLRW cosmology. Following the definition (95) the following commutation relation then holds [3]

$$
\partial_{t}\langle\Psi\rangle_{\mathcal{D}}-\left\langle\partial_{t} \Psi\right\rangle_{\mathcal{D}}=\langle\Psi \Theta\rangle_{\mathcal{D}}-\langle\Psi\rangle_{\mathcal{D}}\langle\Theta\rangle_{\mathcal{D}}
$$

which yields for the expansion scalar $\Theta$

$$
\partial_{t}\langle\Theta\rangle_{\mathcal{D}}-\left\langle\partial_{t} \Theta\right\rangle_{\mathcal{D}}=\left\langle\Theta^{2}\right\rangle_{\mathcal{D}}-\langle\Theta\rangle_{\mathcal{D}}^{2}
$$

Introducing the dimensionless scale factor $a_{\mathcal{D}} \equiv$ $\left(V_{\mathcal{D}} / V_{\mathcal{D} i}\right)^{1 / 3}$ normalized by the volume of the domain $\mathcal{D}$ at some initial time $t_{i}$, we can average the scalar Einstein equations (93a), (93b) and the continuity equation to obtain [3]

$$
\begin{gathered}
\partial_{t}\langle\rho\rangle_{\mathcal{D}}=-\langle\Theta\rangle_{\mathcal{D}}\langle\rho\rangle_{\mathcal{D}} \quad ; \quad\langle\Theta\rangle_{\mathcal{D}}=3 \frac{\partial_{t} a_{\mathcal{D}}}{a_{\mathcal{D}}} \\
\left(\frac{\partial_{t} a_{\mathcal{D}}}{a_{\mathcal{D}}}\right)^{2}=\frac{8 \pi G}{3}\langle\rho\rangle_{\mathcal{D}}-\frac{1}{6}\left(\mathcal{Q}_{\mathcal{D}}+\langle\mathcal{R}\rangle_{\mathcal{D}}\right) \\
\left(\frac{\partial_{t}^{2} a_{\mathcal{D}}}{a_{\mathcal{D}}}\right)=-\frac{4 \pi G}{3}\langle\rho\rangle_{\mathcal{D}}+\frac{1}{3} \mathcal{Q}_{\mathcal{D}}
\end{gathered}
$$

Here, the 'kinematical backreaction' $\mathcal{Q}_{\mathcal{D}}$ is given by

$$
\mathcal{Q}_{\mathcal{D}} \equiv \frac{2}{3}\left(\left\langle\Theta^{2}\right\rangle_{\mathcal{D}}-\langle\Theta\rangle_{\mathcal{D}}^{2}\right)-2\left\langle\sigma^{2}\right\rangle_{\mathcal{D}}
$$

and is a spatial constant over the domain $\mathcal{D}$. 
A necessary condition for (98c) to integrate to (98b) takes the form of the following differential equation involving $\mathcal{Q}_{\mathcal{D}}$ and $\langle\mathcal{R}\rangle_{\mathcal{D}}$,

$$
\partial_{t} \mathcal{Q}_{\mathcal{D}}+6 \frac{\partial_{t} a_{\mathcal{D}}}{a_{\mathcal{D}}} \mathcal{Q}_{\mathcal{D}}+\partial_{t}\langle\mathcal{R}\rangle_{\mathcal{D}}+2 \frac{\partial_{t} a_{\mathcal{D}}}{a_{\mathcal{D}}}\langle\mathcal{R}\rangle_{\mathcal{D}}=0
$$

The equations above describe the essence of Buchert's averaging formalism, for the dust case. We note that the remaining eight Einstein equations for the inhomogeneous geometry, which are not scalar equations, are not averaged. These are the five evolution equations for the trace-free part of the shear,

$$
\partial_{t}\left(\sigma_{B}^{A}\right)=-\Theta \sigma_{B}^{A}-\mathcal{R}_{B}^{A}+\frac{2}{3} \delta_{B}^{A}\left(\sigma^{2}-\frac{1}{3} \Theta^{2}+8 \pi G \rho\right) .
$$

and the three equations relating the spatial variation of the shear and the expansion,

$$
\sigma_{B \| A}^{A}=\frac{2}{3} \Theta_{\| B}
$$

Here, $\mathcal{R}_{B}^{A}$ is the spatial Ricci tensor and, in Buchert's notation, a || denotes covariant derivative with respect to the 3-metric.

In analogy with the dust case, Buchert's averaging formalism can be applied to the case of a perfect fluid [4], by starting from the metric

$$
d s^{2}=-N^{2} d t^{2}+b_{A B} d x^{A} d x^{B} .
$$

The averaged scalar Einstein equations for the scale factor $a_{\mathcal{D}}$ are

$$
\begin{gathered}
3 \frac{\partial_{t}^{2} a_{\mathcal{D}}}{a_{\mathcal{D}}}+4 \pi G\left\langle N^{2}(\rho+3 p)\right\rangle_{\mathcal{D}}=\overline{\mathcal{Q}}_{\mathcal{D}}+\overline{\mathcal{P}}_{\mathcal{D}}, \quad \\
6 H_{\mathcal{D}}^{2}-16 \pi G\left\langle N^{2} \rho\right\rangle_{\mathcal{D}}=-\overline{\mathcal{Q}}_{\mathcal{D}}-\left\langle N^{2} \mathcal{R}\right\rangle_{\mathcal{D}} ; H_{\mathcal{D}}=\frac{\partial_{t} a_{\mathcal{D}}}{a_{\mathcal{D}}},
\end{gathered}
$$

where the kinematical backreaction $\overline{\mathcal{Q}}_{\mathcal{D}}$ is given by

$$
\overline{\mathcal{Q}}_{\mathcal{D}}=\frac{2}{3}\left(\left\langle(N \Theta)^{2}\right\rangle_{\mathcal{D}}-\langle N \Theta\rangle_{\mathcal{D}}^{2}\right)-2\left\langle N^{2} \sigma^{2}\right\rangle_{\mathcal{D}}
$$

and the dynamical backreaction $\overline{\mathcal{P}}_{\mathcal{D}}$ is given by

$$
\overline{\mathcal{P}}_{\mathcal{D}}=\left\langle N^{2} \mathcal{A}\right\rangle_{\mathcal{D}}+\left\langle\Theta \partial_{t} N\right\rangle_{\mathcal{D}},
$$

where $\mathcal{A}=\nabla_{j}\left(u^{i} \nabla_{i} u^{j}\right)$ is the 4-divergence of the 4acceleration of the fluid. Eqn. (105) follows as an integral from Eqn. (104) if and only if the relation

$$
\begin{aligned}
\partial_{t} \mathcal{Q}_{\mathcal{D}} & +6 H_{\mathcal{D}} \mathcal{Q}_{\mathcal{D}}+\partial_{t}\left\langle N^{2} \mathcal{R}\right\rangle_{\mathcal{D}}+2 H_{\mathcal{D}}\left\langle N^{2} \mathcal{R}\right\rangle_{\mathcal{D}}+4 H_{\mathcal{D}} \overline{\mathcal{P}}_{\mathcal{D}} \\
& -16 \pi G\left[\partial_{t}\left\langle N^{2} \rho\right\rangle_{\mathcal{D}}+3 H_{\mathcal{D}}\left\langle N^{2}(\rho+p)\right\rangle_{\mathcal{D}}\right]=0
\end{aligned}
$$

is satisfied. There are also the unaveraged equations (which we do not display here) for the shear, analogous to the shear equations (101) and (102) for dust.
Buchert's approach is the only other approach, apart from Zalaletdinov's MG, which is capable of treating inhomogeneities in a nonperturbative manner, although it is limited to using only scalar quantities within a chosen $3+1$ splitting of spacetime. Buchert takes the trace of the Einstein equations in the inhomogeneous geometry, and averages these inhomogeneous scalar equations. In the context of Zalaletdinov's MG however, we have used the existence of the vector field $\bar{v}^{a}$ in the FLRW spacetime to construct scalar equations after averaging the full Einstein equations. As far as observations are concerned, it has been noted by Buchert and Carfora [17], that the spatially averaged matter density $\langle\rho\rangle_{\mathcal{D}}$ defined by Buchert is not the appropriate observationally relevant quantity - the "observed" matter density (and pressure) is actually defined in a homogeneous space. Since we have done precisely this in Eqn. (45), we are directly dealing with the appropriate observationally relevant quantity in the MG framework.

Another important difference between the two approaches is the averaging operation itself. Buchert's spatial average, defined for scalar quantities, is given (for some scalar $\left.\Psi\left(t, x^{A}\right)\right)$ by (95) above. On the other hand the averaging operation we have been using (given by Eqn. (53) using the volume preserving gauge) is a limit of a spacetime averaging defined using the coordination bivector $\mathcal{W}_{j}^{a^{\prime}}$, and is different from the one in Eqn. (95).

Most importantly though, Buchert's averaging scheme by itself does not incorporate the concept of an averaged manifold $\overline{\mathcal{M}}$ (although the work of Buchert and Carfora [17] does deal with 3-spaces of constant curvature). In a recent paper [20] we had argued that Buchert's "effective scale factor" $a_{\mathcal{D}}(t) \equiv\left(V_{\mathcal{D}}(t) / V_{\mathcal{D}}\left(t_{i n}\right)\right)^{1 / 3}$ must be the scale factor for the metric of the averaged manifold, upto some corrections arising due to such effects as calculated by Buchert and Carfora. In the present work however, it is clear that such a suggestion is necessarily incomplete due to the presence of Eqns. (777) constraining the underlying geometry. These constraints are in general nontrivial and hence indicate that it is not sufficient to assume that the metric of the inhomogeneous manifold averages out to the FLRW form - there are additional conditions which the correlations must satisfy.

To our understanding, Buchert's averaging formalism is a valid aproach, even though it is based on a spatial averaging. A central difference from the MG approach is the issue of closure : not all the Einstein equations have been averaged in Buchert's approach, but only the scalar ones. This puts a constraint on the allowed solutions considered for the averaged equations: (98) for the dust case, and (104) and (105) for the fluid case. Solutions to these equations must necessarily be checked for consistency with the unaveraged equations for the shear. Further, averaging over successively larger scales can bring in additional corrections to the averaged equations, as discussed by Buchert and Carfora. Also, if one does not wish to identify Buchert's $a_{\mathcal{D}}$ with the scale factor in FLRW cosmology, one is compelled to develop a 
whole new set of ideas in order to try and compare theory with observation. On the other hand, if one does identify $a_{\mathcal{D}}$ with the scale factor, comparison with standard cosmology becomes more convenient, but this brings in additional constraints on the underlying inhomogeneous geometry. Thus our conclusion is that the Buchert formalism is a correct and tractable averaging scheme, provided all the caveats pointed out in this paragraph are taken care of. Also, when these caveats have been taken care of correctly, the Buchert formalism is expected to give the same physical results as the MG approach. We recall that in the covariant MG approach also, once a spacetime geometry has been identified for the averaged manifold $\mathcal{M}$, a gauge must be selected for the geometry on the underlying manifold, in order to explicitly compute the correction scalars for comparison with observation.

The advantage of the MG approach is that it accomplishes in a neat package what the Buchert approach, with its attendant caveats, sets out to do. In the MG approach, there are no unaveraged shear equations, because the trace of the Einstein equations has been taken after performing the averaging on the underlying geometry. Since the averaged geometry is FLRW, the shear is zero by definition. There is a natural metric on the averaged manifold by construction, the FLRW metric. The correlations satisfy additional constraints, given by Eqns. (77). Thus, once a gauge has been chosen and if one can overcome the computational complexity of the averaging operation, the cosmological equations derived by us in the MG approach are complete and ready for application, without any further caveats.

In spite of these differences, our equations (81) and (83) for the volume preserving gauge are strikingly similar to Buchert's effective FLRW equations and their integrability condition in the dust case; and in the case of general $N$, the role of Buchert's dynamical backreaction $\overline{\mathcal{P}}_{\mathcal{D}}$ in Eqns. (104) and (108) is identical to that of our combination of $\left(\tilde{\mathcal{P}}^{(2)}+\tilde{\mathcal{S}}^{(2)}\right)$ in Eqns. $87 \mathrm{~b}$ and (90). Concentrating on the volume preserving case, the structure of the correlation $\mathcal{Q}^{(1)}$ is identical to Buchert's kinematical backreaction $\mathcal{Q}_{\mathcal{D}}$ (or $\overline{\mathcal{Q}}_{\mathcal{D}}$ in the general case). The correlation $\mathcal{S}^{(1)}$ appears in place of the averaged 3Ricci scalar $\langle\mathcal{R}\rangle_{\mathcal{D}}$ in Buchert's dust equations. This is not unreasonable since Buchert's $\langle\mathcal{R}\rangle_{\mathcal{D}}$ can be thought of as $\langle\mathcal{R}\rangle_{\mathcal{D}}=6 k_{\mathcal{D}} / a_{\mathcal{D}}^{2}+$ corrections, where $6 k_{\mathcal{D}} / a_{\mathcal{D}}^{2}$ represents the 3-Ricci scalar on the averaged manifold which in our case is zero, and hence $\mathcal{S}^{(1)}$ represents the corrections due to averaging. Further, these similarities are in spite of the fact that our correlations were defined assuming that a volume preserving gauge averages out to the FLRW 3-metric in standard form, whereas Buchert's averaging is most naturally adapted to beginning with a synchronous gauge. This remarkable feature, at least to our understanding, does not seem to have any deeper meaning - it simply seems to arise from the structure of the Einstein equations themselves, together with our assumption $\mathbf{D}_{\bar{\Omega}} \mathbf{Z}^{a}{ }_{b j}{ }_{j}=0$. In the absence of this lat- ter condition, one would have to consider the correlation 3 - and 4 -forms mentioned earlier, and the structure of the correlation terms and their "conservation" equations would be far more complicated.

An entirely different outlook towards his approach has been emphasized to us by Buchert [22]. According to Buchert, the absence of an averaged manifold $\overline{\mathcal{M}}$ is not to be thought of as a 'caveat', but as a feature deliberately retained 'on purpose'. The actual inhomogeneous Universe is regarded by Buchert as the only fundamental entity, and the introduction of an averaged Universe is in fact regarded as an unphysical and unnecessary approximation. As we mentioned earlier, this is probably the most important difference between MG and Buchert's approach. In the latter, contact with observations is to be made by constructing averaged quantities, such as the scalars defined earlier in this section, and by introducing the expansion factor $a_{\mathcal{D}}$. The assertion here is that the averaging of geometry, as discussed in MG or in the Renormalization Group approach of Buchert and Carfora [17] is not an indispensable step in comparing the inhomogeneous Universe with actual observations. The need for averaging of geometry is to be physically separated from simply looking at effective properties (such as the constructed scalars) which can be defined for any inhomogeneous metric. Averaging of geometry becomes relevant if (i) an observer insists on interpreting the data in a FLRW template model, so that (s)he needs a mapping from the actual inhomogeneous slice and its average properties to the corresponding properties in this template, or (ii) one desires a mock metric, to sort of have a thermodynamic effective metric to approximate the real one. (In this context it should perhaps also be mentioned that the importance of a thin time-slice approximation of spacetime averaging (as opposed to a strict spatial averaging) has been stressed also by Buchert [3].)

\section{DISCUSSION}

In this paper we have addressed the issue of modifications to the standard cosmological equations arising out of explicitly accounting for the averaging procedure that must necessarily be performed when studying Cosmology. While this issue has been dealt with in the literature in various forms, the effect of such modifications is still a subject of debate, mainly due to ambiguities in the averaging schemes available. We have applied the formalism of Zalaletdinov's Macroscopic Gravity (MG) which is a fully covariant and nonperturbative averaging scheme, in an attempt to construct gauge independent corrections to the standard FLRW equations.

We find that one cannot escape the problem of gauge dependence entirely, which is mainly due to the fact that Weyl's postulate which is commonly used in the standard approach to Cosmology, must be refined in the context of averaging in General Relativity, and one is forced to assume that the FLRW spatial sections in their natu- 
ral coordinates arise from averaging the inhomogeneous metric in a particular gauge. However, the choice of this gauge can at least formally be left unspecified, and spacetime scalar corrections to the FLRW equations can be constructed. This partially removes the criticism usually faced by adherents of the averaging approach, which essentially states that effects of averaging are likely to be gauge artifacts. We have shown that these effects are independent of the choice of coordinates in the averaged manifold, and depend on one gauge choice - a choice which itself is fundamental to the assumption of large scale homogeneity and isotropy.

One issue which we did not address was the fact that the scale of averaging is likely to be different at different epochs. The scale $L_{\mathrm{FLRW}} \sim 100 h^{-1} \mathrm{Mpc}$ which we mentioned in Section III, would be appropriate for the present day Universe. For the early Universe however, this scale will probably be very different. Nevertheless, since the formalism simply assumes the existence of a scale at which homogeneity sets in, without being affected by its actual value, the equations we have derived within the MG framework will apply to both the present day as well as to the early Universe, albeit with different averaging scales. If one assumes that the length scale of averaging varies slowly compared to the Hubble expansion scale, then it would be appropriate to apply the formalism independently to say, Supernovae data and the last scattering epoch. However, this issue needs to be dealt with more precisely than discussed here.

This brings us to the most important question to be addressed : How does one apply this formalism without knowing the "true" inhomogeneous metric of the Universe? One possible line of attack may be as follows : In the early Universe at least, it might be reasonable to assume that the "true" metric is a perturbation around the FLRW metric. Using some standard gauge, making the necessary assumptions and ensuring that all required conditions are satisfied, one should then be able to explicitly compute the scalar corrections given the initial power spectrum of the fluctuations. The behaviour of these corrections (in the perturbative regime) may yield some insight as to how the corrections might evolve in the present epoch (nonlinear regime). One could then construct reasonable models for the functions $\tilde{\mathcal{P}}^{(1)}, \tilde{\mathcal{S}}^{(1)}$, $\tilde{\mathcal{P}}^{(2)}$ and $\tilde{\mathcal{S}}^{(2)}$ defined in Section IVB. Note that in this regime, one needn't worry about the explicit construction of these quantities - what one needs are physically reasonable models of the time evolution of these quantities (see, e.g., Ref. [21]). We hope to commence such a program in the near future.

\section{Acknowledgments}

We would like to thank Naresh Dadhich, Friedrich Hehl, Claus Kiefer, Alok Maharana, Yuri Obukhov, Sarang Sane, Barbara Sandhofer and Rakesh Tibrewala for useful discussions. It is also a pleasure to acknowledge in- sightful comments from Thomas Buchert, Alan Coley, Syksy Räsänen and Roustam Zalaletdinov on an earlier version of this paper.

\section{APPENDIX A}

In this appendix we give proofs of several results that were used in the text.

\section{Analysis of $\mathbf{D}_{\bar{\Omega}} \bar{g}^{a b}=0$}

We start with the metric

$$
{ }^{(\mathcal{M})} d s^{2}=g_{00}(t, \mathbf{x}) d t^{2}+g_{A B}(t, \mathbf{x}) d x^{A} d x^{B},
$$

on $\mathcal{M}$ and assume that it averages out to the FLRW form (Eqn. (48)):

$$
\begin{aligned}
& G_{00}=\left\langle\widetilde{g}_{00}\right\rangle=-f^{2}(t) ; G_{0 A}=\left\langle\widetilde{g}_{0 A}\right\rangle=0 ; \\
& G_{A B}=\left\langle\widetilde{g}_{A B}\right\rangle=a^{2}(t) \delta_{A B} .
\end{aligned}
$$

We will analyze the second relation of Eqn. (31) and show that it leads to the result $U^{i j} \equiv \bar{g}^{i j}-G^{i j}=0$, where $\overline{\boldsymbol{\Omega}}^{a}{ }_{b}$ refers to the connection 1-forms associated with $G_{i j}$. For this section we use the notation $H=(1 / a)(d a / d t)$. We have

$$
\mathbf{d} \bar{g}^{a b}+\overline{\mathbf{\Omega}}^{a}{ }_{j} \bar{g}^{j b}+\overline{\mathbf{\Omega}}^{b}{ }_{j} \bar{g}^{a j}=0 .
$$

Consider the three cases $(a=b=0),(a=0, b=B)$ and $(a=A, b=B)$ in turn. From the first case $(a=b=0)$ we can conclude that

$$
\begin{gathered}
\bar{g}^{00}(t, \mathbf{x})=-\frac{k(\mathbf{x})}{f^{2}(t)}, \\
\partial_{A} k(\mathbf{x})=2 a^{2} H \delta_{A B} \bar{g}^{0 B} .
\end{gathered}
$$

where $k(\mathbf{x})$ is a positive definite function (so that the metric signature is preserved) which arises as an integration constant and is constrained by Eqn. (A4b). The second case $(a=0, b=B)$ leads to

$$
\begin{gathered}
\bar{g}^{0 B}=\frac{m^{B}(\mathbf{x})}{a(t) f(t)}, \\
\frac{1}{a f} \partial_{J} m^{B}(\mathbf{x})+\frac{a^{2}}{f^{2}} H \delta_{A J} \bar{g}^{A B}-\frac{k(\mathbf{x})}{f^{2}} H \delta_{J}^{B}=0 .
\end{gathered}
$$

where $m^{B}(\mathbf{x})$ is a 3 -vector that arises as a constant of integration like $k(\mathbf{x})$, and is constrained by Eqn. (A5b). Finally, the last case $(a=A, b=B)$ leads to

$$
\bar{g}^{A B}=\frac{1}{a^{2}(t)} s^{A B}(\mathbf{x}),
$$




$$
\frac{1}{a^{2}} \partial_{J} s^{A B}(\mathbf{x})+\frac{1}{a f} H\left(\delta_{J}^{A} m^{B}(\mathbf{x})+\delta_{J}^{B} m^{A}(\mathbf{x})\right)=0 .
$$

Here $s^{A B}(\mathbf{x})$ is another constant of integration, a symmetric 3 -tensor. It can be easily argued that since $f$ and $a$ are not a priori related, both sides of Eqn. (A4b must vanish, which immediately tells us that the vector $m^{B}(\mathbf{x})$ must vanish, and the function $k(\mathbf{x})$ must be a constant,

$$
k(\mathbf{x})=k=\text { constant } ; m^{B}(\mathbf{x})=0 .
$$

Equations (A5b) and (A6b) then give us

$$
s^{A B}(\mathbf{x})=k \delta^{A B},
$$

with the same constant $k$ as in Eqn. (A7). Finally, putting everything together we find

$$
\begin{gathered}
\bar{g}^{00}=-\frac{k}{f^{2}} ; \quad \bar{g}^{0 A}=0 \quad ; \quad \bar{g}^{A B}=\frac{k}{a^{2}} \delta^{A B}, \\
\Rightarrow \bar{g}^{i j}=k G^{i j} .
\end{gathered}
$$

The constant $k$ is not constrained by any of the equations and appears to be a free parameter in the theory. The modified Einstein equations (35) show that $k$ can be absorbed into the averaged energy momentum tensor. We will for simplicity assume $k$ to be unity thereby obtaining, as required

$$
U^{i j} \equiv \bar{g}^{i j}-G^{i j}=0 .
$$

\section{Analysis of the condition $\left\langle\Gamma_{b c}^{a}\right\rangle={ }^{(\mathrm{FLRW})} \Gamma_{b c}^{a}$}

Here we will assume that the line element on $\mathcal{M}$ is in the volume preserving gauge

$$
{ }^{(\mathcal{M})} d s^{2}=-\frac{d \bar{t}^{2}}{h(\bar{t}, \mathbf{x})}+h_{A B}(\bar{t}, \mathbf{x}) d x^{A} d x^{B},
$$

so that the averaging is trivial, and the metric and averages out to the FLRW line element on $\overline{\mathcal{M}}$ given by

$$
{ }^{(\overline{\mathcal{M}})} d s^{2}=-\frac{d \bar{t}^{2}}{\langle h\rangle(\bar{t})}+\bar{a}^{2}(\bar{t}) \delta_{A B} d x^{A} d x^{B},
$$

where we used the condition $\langle 1 / h\rangle=1 /\langle h\rangle$ that follows from $\bar{g}^{00}=G^{00}$. The conditions $\left\langle\Gamma_{b c}^{a}\right\rangle={ }^{(\text {FLRW })} \Gamma_{b c}^{a}$ then result in the following :

$$
\begin{array}{ll}
\Gamma_{00}^{0}:\left\langle\partial_{\bar{t}}(\ln \sqrt{h})\right\rangle=\partial_{\bar{t}}(\ln \sqrt{\langle h\rangle}), \\
\Gamma_{0 A}^{0}:\left\langle\partial_{A}(\ln \sqrt{h})\right\rangle=0, \\
\Gamma_{00}^{A}:\left\langle\frac{h^{A B}}{h} \partial_{B}(\ln \sqrt{h})\right\rangle=0, \\
\Gamma_{0 B}^{A}:\left\langle\frac{1}{\sqrt{h}} \Theta_{B}^{A}\right\rangle=H \delta_{B}^{A}, \\
\Gamma_{A B}^{0}:\left\langle\sqrt{h} \Theta_{A B}\right\rangle=\langle h\rangle \bar{a}^{2} H \delta_{A B}, \\
\Gamma_{B C}^{A}:\left\langle{ }^{(3)} \Gamma_{B C}^{A}\right\rangle=0 .
\end{array}
$$

Eqns. (A13b) and (A13f) are consistent with each other since ${ }^{(3)} \Gamma_{B A}^{A}=\partial_{B}(\ln \sqrt{h}$ ), and Eqn. (A13C) is consistent with the assumption Eqn. (30a). The trace of Eqn. (A13d) gives $\langle(1 / \sqrt{h}) \Theta\rangle=3 H$. However, using Eqn. (61) we have $(1 / \sqrt{h}) \Theta=N \Theta=\partial_{\bar{t}}(\ln \sqrt{h})$, and combined with Eqn. (A13a) this gives

$$
\frac{1}{2} \partial_{\bar{t}}(\ln \langle h\rangle)=3 \partial_{\bar{t}}(\ln \bar{a}) \Rightarrow\langle h\rangle=\bar{a}^{6}
$$

where we have set an arbitrary proportionality constant (representing rescaling of the time coordinate by a constant) to unity. This establishes the last equality in Eqn. (55).

Finally, consider the trace $\left(\left\langle h^{A B}\right\rangle /\langle h\rangle\right)\left\langle\sqrt{h} \Theta_{A B}\right\rangle$ : using the condition $\bar{g}^{A B}=G^{A B}$, Eqn. (A13e) and the trace of Eqn. (A13d), this gives us

$$
\begin{aligned}
\frac{\left\langle h^{A B}\right\rangle}{\langle h\rangle}\left\langle\sqrt{h} \Theta_{A B}\right\rangle & =\frac{1}{\langle h\rangle} \frac{\delta^{A B}}{\bar{a}^{2}}\left\langle\sqrt{h} \Theta_{A B}\right\rangle \\
& =3 H=\left\langle\frac{1}{\sqrt{h}} \Theta\right\rangle=\left\langle\frac{h^{A B}}{h}\left(\sqrt{h} \Theta_{A B}\right)\right\rangle .
\end{aligned}
$$

On using the condition (30a) this leads to

$$
\left(\frac{\left\langle h^{A B}\right\rangle}{\langle h\rangle}-\left\langle\frac{h^{A B}}{h}\right\rangle\right)\left\langle\Gamma_{A B}^{0}\right\rangle=0,
$$

which is consistent with the assumption

$$
\frac{\left\langle h^{A B}\right\rangle}{\langle h\rangle}=\left\langle\frac{h^{A B}}{h}\right\rangle .
$$


[1] G F R Ellis, in General Relativity and Gravitation (D. Reidel Publishing Co., Dordrecht), Eds. B. Bertotti et al., (1984).

[2] T W Noonan, Gen. Rel. and Grav., 16, 1103, (1984); T Futamase, Phys. Rev. Lett., 61, 2175, (1988);

M Kasai, Phys. Rev. Lett., 69, 2330, (1992);

J P Boersma Phys. Rev. D57, 798, (1998) arXiv:gr-qc/9711057;

Several other references and a discussion on the issue of averaging in cosmology can be found in - A Krasiński, Inhomogeneous Cosmological Models, Cambridge Univ. Press, 1997.

[3] T Buchert, Gen. Rel. and Grav., 32, 105, (2000) arXiv:gr-qc/9906015.

[4] T Buchert, Gen. Rel. and Grav., 33, 1381, (2001) arXiv:gr-qc/0102049.

[5] E W Kolb, S Matarrese and A Riotto, submitted to Phys. Rev. Lett. arXiv:hep-th/0503117. (2005); New J. Phys. 8, 322, (2006) arXiv:astro-ph/0506534.

[6] G Geshnizjani, D Chung and N Afshordi, Phys. Rev. D72, 023517, (2005) arXiv:astro-ph/0503553;

E E Flanagan, Phys. Rev. D71, 103521, (2005) arXiv:hep-th/0503202;

C M Hirata and U Seljak, Phys. Rev. D72, 083501, (2005) arXiv:astro-ph/0503582;

A Notari, Mod. Phys. Lett. A21, 2997, (2006) arXiv:astro-ph/0503715.

[7] A Ishibashi and R M Wald, Class. Quant. Grav., 23, 235, (2006) arXiv:gr-qc/0509108.

[8] S Räsänen, JCAP, 0402:003, (2004) arXiv:astro-ph/0311257; JCAP, 0411:010, (2004) arXiv:gr-qc/0408097; Class. Quant. Grav. 23, 1823, (2006) arXiv:astro-ph/0504005]; JCAP, 0611:003, (2006) arXiv:astro-ph/0607626.

[9] R M Zalaletdinov, Gen. Rel. E Grav., 25, 673, (1993).

[10] M Mars and R M Zalaletdinov, J. Math. Phys., 38, 4741, (1997) arXiv:dg-ga/9703002.

[11] R M Zalaletdinov, Gen. Rel. \& Grav., 24, 1015, (1992); Bull. Astron. Soc. India, 25, 401, (1997) arXiv:gr-qc/9703016;

R M Zalaletdinov, to appear in Proceedings of Atlantic Canada General Relativity Regional Meeting,
Fredericton, New Brunswick, Canada, 5-7 May 2006 arXiv:gr-qc/0701116. (2007).

[12] A A Coley, N Pelavas and R M Zalaletdinov, Phys. Rev. Lett., 95, 151102, (2005) arXiv:gr-qc/0504115.

[13] A A Coley and N Pelavas, Phys. Rev. D74, 087301, (2006) arXiv:astro-ph/0606535; Phys. Rev. D75, 043506, (2007) arXiv:gr-qc/0607079.

[14] A A Coley, arXiv:0704.1734], (2007).

[15] We are making the assumption $N^{A}=0$ in the volume preserving gauge for algebraic convenience only - in our case this assumption cannot be justified by the absence of vorticity. A more detailed (and complicated) analysis should retain an arbitrary $N^{A}$ in the inhomogeneous geometry, and make assumptions about its average - such as $\left\langle N_{A}\right\rangle=0$ for example.

[16] In fact, the shape of the spacetime averaging region can also be left arbitrary, provided the spatial averaging limit is correctly taken as the limit in which one is essentially integrating over a spatial domain only. We will not venture into this complication.

[17] T Buchert and M Carfora, Phys. Rev. Lett., 90, 031101, (2003) arXiv:gr-qc/0210045] Class. Quant. Grav., 19, 6109, (2002) arXiv:gr-qc/0210037.

[18] D L Wiltshire, arXiv:gr-qc/0702082 (2007); arXiv:gr-qc/0503099 (2005)

[19] D Palle, Nuovo Cim., 117B, 687, (2002) arXiv:astro-ph/0205462;

J D Barrow and C G Tsagas, Class. Quant. Grav., 24, 1023, (2007) arXiv:gr-qc/0609078.

$\mathrm{K}$ Enqvist and $\mathrm{T}$ Mattson JCAP 0702:019, (2007) arXiv:astro-ph/0609120;

A E Romano, Phys. Rev. D75, 043509, (2007) arXiv:astro-ph/0612002;

N Li and D Schwarz, arXiv:gr-qc/0702043], (2007);

$\mathrm{T}$ Biswas and A Notari, arXiv:astro-ph/0702555, (2007).

[20] A Paranjape and T P Singh, arXiv:astro-ph/0609481 (2006), submitted.

[21] T Buchert, J Larena and J-M Alimi, Class. Quant. Grav., 23, 6379, (2006) arXiv:gr-qc/0606020.

[22] T Buchert, private communication. 